\title{
The influence of parafoveal preprocessing and linguistic context on the optimal landing position effect
}

\author{
FRANCOISE VITU \\ Groupe Regard, Laboratoire de Psychologie Expérimentale, CNRS \\ Université René Descartes, EPHE, EHESS, Paris, France
}

\begin{abstract}
Several experiments have shown the existence of an optimal landing position effect during isolated word recognition as well as during text reading; both the probability of refixating the test word and the gaze duration are smaller if the eye first fixates near the center of the word than if the eye first fixates other positions in the word. However, recent data indicate that the optimal landing position effect is weakened under normal reading conditions, when words are included in texts. The purpose of the present experiment was to test whether parafoveal preprocessing or linguistic context specific to the text reading situation could be responsible for this weakening. With a paradigm that approximates normal reading, albeit in a somewhat slower manner, it was shown that although the possibility of preprocessing words in parafoveal vision and of predicting them from preceding context globally affected refixation probability and gaze duration on the word, this did not strongly affect the optimal landing position effect. Since the global effects of these factors were comparable to those found by other authors in normal reading, it was concluded that the weakening of the optimal landing position effect during text reading results from the influence of other factors peculiar to this situation. Hypotheses are proposed as explanations of the effects of the manipulated factors on oculomotor behavior.
\end{abstract}

In a number of recent experiments, the existence of an optimal landing position in words has been shown during isolated word recognition (Holmes \& O'Regan, 1987; O’Regan, 1984, 1989; O'Regan \& Lévy-Schoen, 1987; O'Regan, Lévy-Schoen, Pynte, \& Brugaillère, 1984). In these experiments the eye's initial fixation position in the word was manipulated, and it was observed that if the eye first fixated near the middle of the word, the probability of refixating the word and, consequently, the gaze duration (the total time the eye spent on the word) were smaller than they were when the eye first fixated other positions in the word. Individual fixation durations were also shown to depend on the eye's first fixation position in the word as well as on within-word tactics (whether one, two, or more fixations were made within the word).

On the basis of these results, O'Regan and Lévy-Schoen (1987) and O'Regan (1990) have suggested that withinword tactics during text reading may be determined by a preestablished program based on the eye's initial landing position. That is, if the eye lands in a region that is generally optimal (near the word's middle), the eye would directly leave the word, and, on the contrary, if the eye lands at a nonoptimal position, it would refixate the word before leaving it. Individual fixation durations would de-

I wish to thank Kevin O'Regan, Keith Rayner, Albrecht Inhoff, and Paul Kerr fo' helpful comments. Correspondence should be addressed to Françoise Vitu, Groupe Regard, Laboratoire de Psychologie Expérimentale, CNRS, Université René Descartes, EPHE, EHESS, 28 rue Serpente, 75006 Paris, France. pend on the tactics adopted on the word and also on the eye's first fixation position in the word.

Results from recent experiments seem to favor this hypothesis, since they have confirmed the existence of an optimal landing position for words included in texts (McConkie, Kerr, Reddix, Zola, \& Jacobs, 1989; Vitu, O'Regan, \& Mittau, 1990). However, a direct comparison of the optimal landing position curves obtained during isolated word and text reading (see Figure 1) has shown that the optimal landing position phenomenon is weakened in text reading (Vitu et al., 1990). Thus, whereas during isolated word recognition, within-word tactics and gaze durations are primarily determined by the eye's initial landing position, it seems that during text reading, they result not only from the effect of this factor but also from its interaction with factors specific to text reading. The present experiment represents an attempt to identify the additional factors that interact with the eye 's initial landing position and that might be responsible for the weakening of the optimal landing position phenomenon during text reading.

A major difference between isolated word and text reading is that during text reading, words are generally predictable from preceding context and are available in parafoveal vision before their fixation. Such factors have been shown to facilitate word recognition and also to affect eye movement behavior toward words. The naming latency of a word is shorter when an item presented before the word in parafoveal vision is identical to the word than it is when the item is different (Balota \& Rayner, 1983; McClelland 


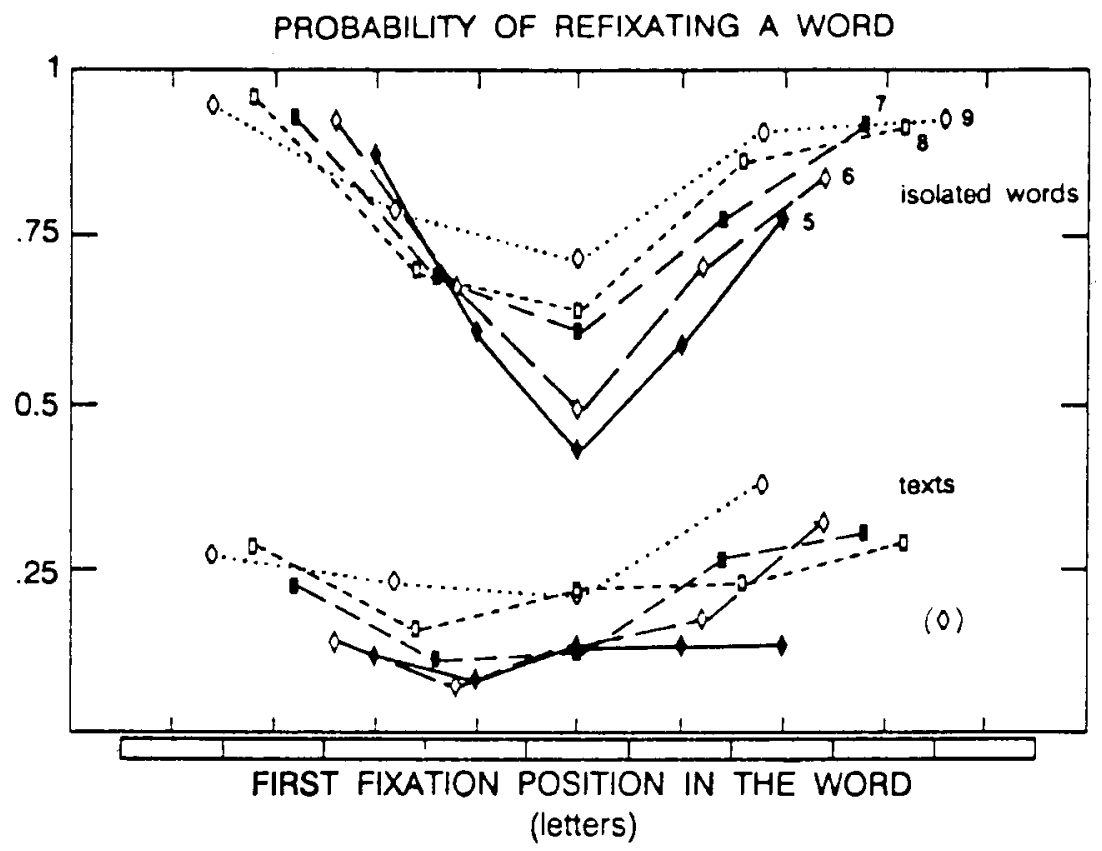

Figure 1. The optimal landing position effect obtained by Vitu, ORegan, and Mittau (1990) during isolated word recognition and text reading. The graph represents the probability of refixating the test word as a function of the eye's initial landing position in the test word, for words of $5,6,7,8$, and 9 letters, in both isolated word recognition (upper curves) and text reading (bottom curves). For each length, data are plotted relative to the middle of the word. The blocks below the abscissa show the positions that the letters of a 9-letter word would occupy.

\& O’Regan, 1981; Rayner, 1978; Rayner, McConkie, \& Ehrlich, 1978; Rayner, McConkie, \& Zola, 1980). Lexical decision time and naming latency are decreased when a test word is preceded by a word prime or a sentence prime which allows it to be predicted (Balota \& Rayner, 1983; Carroll \& Slowiaczek, 1986; Fischler \& Bloom, 1979, 1985; Meyer \& Schvaneveldt, 1971; Paap \& Newsome, 1981; Schubert \& Eimas, 1977; Schwanenflugel \& Shoben, 1985; Simpson, Peterson, Casteel, \& Burgess, 1989; Stanovich \& West, 1979, 1981, 1983). Moreover, the effect of parafoveal preprocessing is larger for words that are predictable from their preceding context (McClelland \& O'Regan, 1981; Paap \& Newsome, 1981). Within-word tactics as well as gaze durations and individual fixation durations on words are also influenced by parafoveal preprocessing (Balota, Pollatsek, \& Rayner, 1985; Inhoff, 1989; Lima \& Inhoff, 1985; McConkie, Underwood, Zola, \& Wolverton, 1985) and by linguistic context (Ehrlich \& Rayner, 1981; Zola, 1984), the effect of parafoveal preprocessing being stronger for highly predictable words than it is for less predictable words (Balota et al., 1985).

We could suppose that the weakening of the optimal landing position effect during text reading relative to isolated word reading might result from the influence of parafoveal preprocessing or linguistic context, since these could diminish the necessity of first fixating a particular position in the word to ensure efficient processing. In other words, these factors could decrease the effect of the eye's initial landing position on refixation probability and gaze duration.

Another important difference between isolated word and text reading is that in text reading, the eye is engaged in a sequence of forward movements (a reading rhythm), which could decrease the probability of refixating words, whatever the initial landing position in a word might be. Such a factor could also be responsible for the weakening of the optimal landing position effect during text reading.

Vitu and O'Regan $(1988,1990)$ tested the influence of parafoveal preprocessing on the optimal landing position phenomenon. An isolated test word that was either visible or masked appeared in parafoveal vision, followed by a comparison word that was masked until it was fixated. The subject's task was to read the test word and then the comparison word, in order to decide whether the two words were identical. The eye's initial fixation position in the test word was manipulated by shifting the word to either the right or the left during the execution of the saccade toward the test word. Although the results showed a global effect of parafoveal preprocessing on the probability of refixating words, they did not show an influence of parafoveal preprocessing on the strength of the optimal landing position phenomenon.

These results seem to indicate that parafoveal preprocessing cannot be responsible for the weakening of the optimal landing position phenomenon observed during text 
reading. This conclusion was also reached by Vitu et al. (1990), who did an a posteriori analysis of the data obtained during text reading and found that the strength of the optimal landing position effect did not differ as a function of the distance from the test word from which a saccade was launched-which is presumably correlated with the amount of parafoveal preprocessing that can be done. The latter finding seems also to indicate that parafoveal preprocessing, even when it is reinforced by contextual information, does not modify the strength of the optimal landing position phenomenon. However, these data probably contained data for a mixture of cases in which words were either highly predictable or less predictable from their preceding context. Thus, the interactive influence of parafoveal preprocessing and linguistic context on the optimal landing position effect has yet to be tested in a situation in which the linguistic context that precedes the word to be recognized is manipulated. We might also suppose that linguistic context could be the only factor responsible for the weakening of the optimal landing position phenomenon observed in text reading.

The purpose of the present experiment was therefore to test the separate and combined influences of parafoveal preprocessing and linguistic context on the optimal landing position phenomenon. As in Vitu and O'Regan (1988, 1990), an isolated test word was presented either visible or masked in parafoveal vision. This time, however, the appearance of the test word was preceded either by a sentence that strongly constrained the word or by a neutral sentence. To introduce linguistic context, a sentence prime was used instead of a word prime in order more closely to approximate text reading, because Simpson et al. (1989) and Duffy, Henderson, and Morris (1989) have shown that the influence on word recognition of a contextual sentence is due to the whole sentential context and not just to association with the individual words it contains. In the present experiment, the test word was followed by further words that could be either compatible or not compatible with the beginning of the sentence formed by the sentence prime and the test word. The presence of words after the test word ensured similarity with situations in which the optimal landing position phenomenon has been found (O'Regan \& Lévy-Schoen, 1987; O'Regan et al., 1984; Vitu \& O'Regan, 1988, 1990; Vitu et al., 1990), and also with text reading. The subject's task was to read the sentence prime, the test word, and the sequence of remaining words, in order to decide whether the whole sentence (sentence prime + test word + remaining words) was coherent or not.

When the task is to land on isolated words, the spontaneous initial landing position of the eye in words is generally concentrated within a narrow region (Vitu, in press). Since the present purpose was to analyze the oculomotor behavior on words as a function of the eye's first fixation position in each word, the initial landing position in the test word was manipulated by shifting the test word to the left or the right during the execution of the saccade toward the test word. Eye movements during reading of the test word were recorded. The probability of refixating the test word, gaze duration, and individual fixation durations were measured as a function of the eye's initial landing position in the test word, in the different conditions of parafoveal preprocessing and linguistic context.

The reader should note that the constraints imposed by this recording system make the present experimental situation different from normal text reading. The most important difference lies in the delay that elapses between the reading of the inducing sentence and the beginning of the reading of the test word, which is often as much as $500 \mathrm{msec}$ and is thus longer than such delays during normal text reading. It seems reasonable, however, to assume that this delay should reinforce any facilitatory effects of context and/or parafoveal preprocessing by giving them more time to act. Thus, if these factors should be shown to globally affect the refixation probability and the gaze duration and yet not to weaken the strength of the optimal landing position effect, then it would seem justified to suppose that during normal text reading these factors would a fortiori also not be acting to weaken the optimal landing position effect. The weakening of the optimal landing position effect observed in normal reading (Vitu et al., 1990) would thus have to result from the influence of other factors peculiar to reading, such as reading rhythm.

\section{METHOD}

\section{Subjects}

Twenty subjects participated in the experiment. They were between 20 and 30 years old. All were native speakers of French, and all were unfamiliar with the purpose of the experiment.

\section{Materials}

Two lists of 240 test words were constructed. Each contained 120 words with a length of 5 letters and 120 words with a length of 9 letters. For each length, there were 60 test words of low frequency and 60 test words of high frequency, as defined by the Tresor de la langue française count of 38 million words. The median frequencies were approximately equal for the two word lengths; for the first list, the medians for low-frequency words were 1.3 and 1.1 per million (for 5- and 9-letter words, respectively) and the medians for high-frequency words were 95 and 82 per million (for 5- and 9-letter words, respectively); for the second list, the medians for low-frequency words were 1.2 and 1.3 per million (for 5- and 9-letter words, respectively) and the medians for highfrequency words were 93 and 75 per million (for 5- and 9-letter words, respectively).

For each test-word list, two lists of sentence primes were constructed; one list consisted of sentences that permitted the corresponding test word to be easily predicted (e.g., "le marin attend que la tempête se calme" [" "the sailor is waiting for the storm to abate"]), and the other list contained neutral sentences that could induce not only the test word but also many other words (e.g., "Vincent proposa que Pascal se calme" ["Vincent proposed that Pascal be quiet']). For each test word, the two corresponding sentence primes had approximately the same lengths and the same syntactic structures.

The predictability of each of the test words from each sentence prime was tested in a preliminary experiment, in which a list of 
sentence primes was presented on paper to an independent group of subjects. The sentence primes were in half the cases supposed to be predictable and in half the cases supposed to be neutral. However, for each test word, each subject saw only the predictable sentence prime or the neutral sentence prime. Both sentences (predictable and neutral) corresponding to each test word were tested over two independent groups of subjects (20 subjects each). The order in which predictable and neutral sentence primes appeared was randomized and different for each subject. The subjects had to read the sentence prime and to write the word(s) that they thought were induced by the sentence. They were instructed to answer as quickly as possible and to write the words in the order in which they occurred. For the analysis, only the first written word was considered. A sentence prime was considered to be effectively predictable when it induced the test word or a synonymous word in $75 \%$ of the cases. A sentence prime was considered to be neutral when, over all subjects, it induced not only the test word but a variety of other words as well.

For each test-word list, a list of remaining word sequences was constructed. Each sequence of remaining words was chosen in such a way that the whole sentence formed by the sentence prime, the test word, and the sequence of remaining words constituted a coherent sentence or did not. The sequence of remaining words was always syntactically compatible with what preceded it, but in only half of the cases was it semantically coherent with the beginning of the sentence (sentence prime + test word) (e.g., "le marin attend que la tempête se calme pour partir" ["the sailor is waiting for the storm to abbate before leaving''], which is a semantically coherent form, and "il sortit son briquet et alluma une cigarette échancrée" ["he took his lighter and lighted a cigarette that was indented"'], which is not semantically coherent).

Two lists of pairs of isolated letters (test letter + comparison letter) were also constructed, in order to test calibration accuracy during the experiment. The fixation of these letters was always preceded by the following sentence: "Fixez précisément chaque lettre" ("Fixate each letter accurately").

Two lists of 24 training test words and two corresponding lists of sentence primes that were either predictable or neutral were constructed.

When the test word appeared in parafoveal vision in the condition without parafoveal preprocessing, it was masked. The mask was the following: Each letter of the word was modified so that the number of lighted pixels in the matrix defining the masked character was identical to the number in the corresponding unmasked character; the lighted pixels appeared at random positions in the matrix. Thus, the masked words were nonhomogeneous stimuli that resembled words, with the same number of lighted pixels. This kind of masking allowed a close reproduction of the visual aspects of the words so that both conditions of parafoveal preprocessing (with and without) were globally equivalent and differed only locally (at the level of the letters that constituted each word).

\section{Design}

The test words had two lengths (5 and 9 letters) and two frequencies (high and low). Isolated test words were presented in parafoveal vision in two ways: visible or masked. These two conditions of parafoveal preprocessing (with and without) appeared in two different blocks of trials that corresponded to the two lists of test words, respectively. The list of words attributed to each condition of parafoveal preprocessing was counterbalanced over subjects.

The sentence prime that preceded the test word was either predictable or neutral. These two conditions of preceding context were mixed in each block of trials. For one condition of parafoveal preprocessing, and thus for one list of test words, half of the subjects saw the first half of each test word preceded by a predictable sentence and the second half of each test word preceded by a neutral sentence, and the other half of the subjects saw the first half of each test word preceded by a neutral sentence and the second half of each test word preceded by a predictable sentence. Thus, all the subjects saw all the test words, and over all subjects, all the test words were seen preceded by a predictable and neutral sentence prime.

In one block of trials, the order of presentation of the different test words preceded by a sentence prime that was either predictable or neutral was randomized and different for each subject. The order in which subjects passed the two blocks was counterbalanced between subjects; half of the subjects saw the test words in the condition with parafoveal preprocessing first and then in the condition without parafoveal preprocessing, and the other half were tested with the conditions in the opposite order.

The eye's initial fixation position in the test word was manipulated by shifting the test word to the left or the right in different amounts. The amounts of shift were calculated on the basis of data indicating that the mean spontaneous landing position of the eye in isolated words was located on the 2nd or 3rd letter of 5-letter words and on the 3rd letter of 9-letter words (Vitu, in press). The selected shift amounts relative to the beginning of the test word were the following: $+1,0,-1,-2$, and -3 , for 5 -letter words, and +2 , $0,-2,-4$, and -6 , for 9-letter words; a shift of 0 corresponded to no displacement of the test word. Thus, in most cases, the eye would land on Letter 1, 2, 3, 4, or 5 in 5-letter words, and on Letter $1,3,5,7$, or 9 in 9-letter words. The different amounts of shift of the test word were mixed in each block of trials and occurred in a random order.

\section{Procedure}

The subjects sat in an adjustable chair, $50 \mathrm{~cm}$ from the computer screen. A bite bar was used to minimize head movements, since these affected the accuracy of eye movement recording on the test word.

Following a calibration procedure in a 26-character zone $\left(15.6^{\circ}\right)$ in the region where test words could appear, the first trial began (the sequence of events during one trial is described in Figure 2). First an asterisk appeared on the left of the screen. When the eye fixated the asterisk precisely (to within .25 of a character from the center of the asterisk), the asterisk disappeared and the sentence prime appeared, beginning two characters left of where the asterisk had been. The subject read the sentence and then pressed the space bar. Then an asterisk appeared on the right of the screen. If the eye did not fixate within 1.5 characters from the center of this asterisk, a new calibration phase was interposed and the sentence prime was repeated. The verification of the accuracy on this asterisk precluded any loss of trials in which calibration was inaccurate. In the case in which the eye fixated within the accepted error margin, this asterisk disappeared, and the trial continued as follows.

An asterisk was presented on the left side of the screen, simultaneously with the disappearance of the preceding one. If the eye did not fixate the asterisk accurately (to within .25 of a character from the center of the asterisk), a new calibration phase was interposed, and the sentence prime was repeated. If the eye fixated accurately, the asterisk disappeared and a second one simultaneously appeared three characters to the right. When the computer detected a saccade toward this second asterisk, the test word appeared two space characters away from the asterisk, and a string composed of 26 masked characters simultaneously appeared, starting at a position one character from the end of the test word. The test word was visible (in the condition with parafoveal preprocessing) or masked until the eye began to move in its direction (in the condition without parafoveal preprocessing). The string of masked characters placed at the right of the test word indicated the presence of the sequence of remaining words but ensured that the oculomotor behavior on the test word would not be influenced by the variability in the number of words that constituted this sequence. 


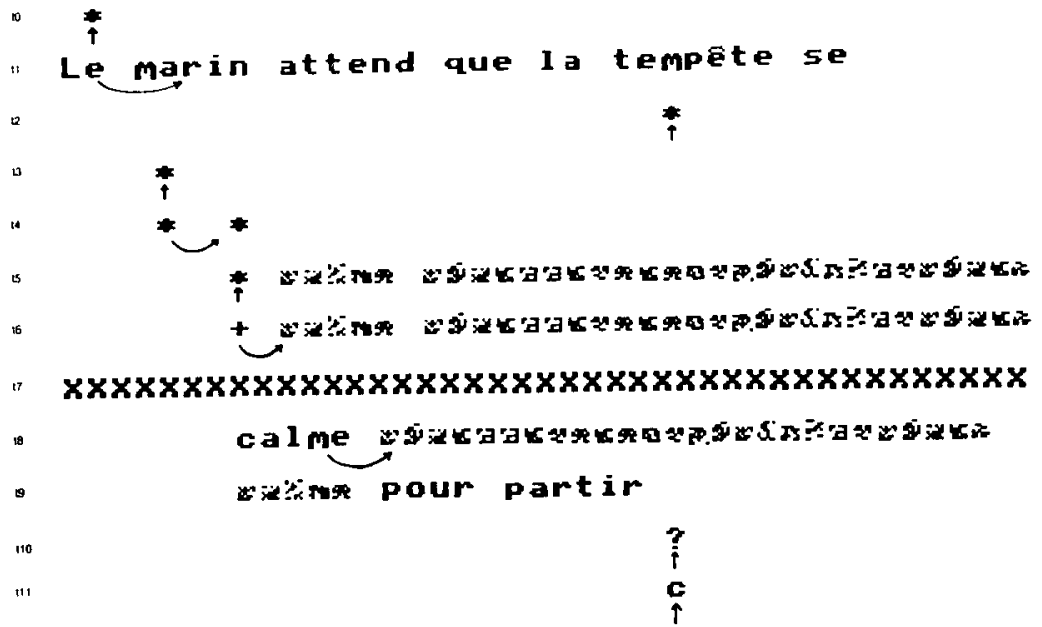

Figure 2. The sequence of events during one trial: The subject has first to fixate an asterisk on the left side of the screen $(\mathbf{t 0})$. When a fixation is detected, the sentence prime appears (t1). The subject has to read this sentence and to press a bar, which makes an asterisk appear on the right side of the screen (t2). When the subject fixates this asterisk, another one appears on the left side of the screen ( $t 3)$. After fixation of this asterisk, a last one appears three characters to its right (t4). During the saccade toward this last asterisk, the test word, which is either visible or masked, appears with a string of masked characters on its right (t5). Note that in this example, the test word appears masked as in the condition without parafoveal preprocessing. The subject has to fixate this last asterisk and then read the test word. When a fixation on the last asterisk is detected, it is transformed into a cross (t6). From the moment the computer detects that the eye is beginning to move toward the test word, a line of Xs is flashed (t7). Then, the test word and the string of masked characters are shifted rightward or leftward by a certain amount (which is variable from one trial to the other), and the test word becomes visible if it has previously been masked (t8). Note that in this example, the amount of shifting is 2 letters to the left. When the eye leaves the test word, the test word is masked and the string of masked characters is transformed into the sequence of remaining words (t9). The subject has to read this sequence of remaining words and then press a bar, which makes a question mark appear (t10)-indicating that the subject must decide whether the whole sentence formed by the sentence prime, the test word, and the remaining words is semantically coherent or not. Pressing a button makes the response appear ("c" or " $n$ "), and this must also be fixated (t11). Then the next trial begins.

If the eye did not accurately fixate the second asterisk (to within 1.5 characters from its center), a new calibration phase was interposed, and for the condition without parafoveal preprocessing, the sentence prime was repeated; for the condition with parafoveal preprocessing, a new trial began. If the computer detected that the eye accurately fixated the second asterisk, this asterisk was immediately transformed into a cross. The subject's task was to saccade toward the test words only when the cross appeared. This procedure, based on the results of Ross and Ross (1980), permitted the delay of the saccade and thus ensured that saccade latencies would be comparable to fixation durations observed during text reading From the moment at which the computer detected that the eye had passed the first of the two spaces separating the cross and the beginning of the test word, the cross disappeared and the test word became visible (in the condition without parafoveal preprocessing). Moreover, at the same time, in both conditions, the test word and the stimulus representing the sequence of remaining words were shifted by $\approx$ variable amount to either the right or the left. In order that both conditions of parafoveal preprocessing and all shift amounts be considered equivalent display conditions, from the moment at which the computer detected the saccade toward the test word, a line of Xs was flashed, which remained visible for $60 \mathrm{msec}$. This delay was too long for the large perturbation not to be visible. What is important, however, is that the visible perturbation was the same for all shifts, rendering negligible any differences caused by the amounts of shift.

The subject read the test word. When the computer detected that the eye had passed the middle of the space separating the test word and the 26-masked-character string, the test word was masked and the sequence of remaining words became visible. When the subject had finished reading this sequence, he or she pressed the space bar, and a question mark appeared on the right side of the screen, indicating that the subject had to decide whether the whole sentence formed by the sentence prime, the test word, and the sequence of remaining words corresponded to a semantically correct sentence. The subject then pressed one of two buttons to indicate the response, and the computer materialized the choice by displaying a " $c$ " for coherent or an " $n$ " for noncoherent. The subject was instructed to check the response by fixating this letter (which the subjects did naturally anyway). The accuracy of this fixation permitted the calibration accuracy to be checked. If the eye fixated to within 2 characters from the response letter, the data for the trial were kept and a linear interpolation between the fixated position for this asterisk and the fixated position for the asterisk that preceded the saccade into the test word was made on the data. Then the next trial began. If the eye did not fixate the response to within 2 characters, the 
data for the trial were not kept, and a new calibration phase was interposed before the next trial.

It must be noted that throughout the process of scanning the asterisks, the time that it took the computer to detect a fixation was $30-60 \mathrm{msec}$, and the time it took to detect an eye position change was $10 \mathrm{msec}$, which are very short delays. The time that elapsed between the end of the sentence prime reading and the beginning of the test word reading was thus of the order of 500-700 msec.

On every 10th trial, a pair of isolated letters was presented instead of a test word (at 7 and 17 characters from the last asterisk, respectively). The presentation of these isolated letters was preceded by the presentation of the sentence "Fixez précisément chaque lettre" ("Fixate each letter accurately"). The procedure that preceded the fixation of the isolated letters was exactly the same as that used for test words, except that the isolated letters were not shifted. Each letter was masked until the eye fixated it. The subject's task was to fixate the two isolated letters successively and then to decide whether they were identical.

The two experimental blocks of trials were preceded by the two training blocks of 24 trials.

\section{Apparatus}

Eye movements were monitored by a photoelectric, infrared, iris/sclera boundary detection technique. Eye position was sampled by a BBC computer every $10 \mathrm{msec}$. Saccade size and fixation duration were analyzed by computer-a saccade was defined as a change in eye position of more than half a character, taking less than $30 \mathrm{msec}$ and giving rise to a fixation lasting more than 30-60 msec. In both conditions (isolated word and text reading), stimuli were presented on a black and white Velec (1614-07) video monitor, refreshed every $20 \mathrm{msec}$. Characters were defined in an $8 \times 8$ pixel matrix that subtended $.6^{\circ}$.

\section{RESULTS}

The recording accuracy was verified by analyzing fixation positions on the first isolated letter. This analysis revealed that the accuracy was better than \pm .5 letter. The analysis of the oculomotor behavior on test words concerned only cases for which the given answer (coherent or not) was correct, since a bad answer could be attributable to the fact that the subject did not correctly read the sentence prime and/or the test word. However, the percent of incorrect responses was low (less than 10\%). Only cases for which the sentence prime was presented no more than twice were kept. It has to be noted that they were only few cases for which the sentence prime was repeated (7\%). Cases for which individual fixation durations were less than $70 \mathrm{msec}$ were excluded from the analysis.

\section{The Effect of the Shifting}

of the Test Word

A first analysis concerned the initial landing position in the test word as a function of the amount of shift of the test word. This analysis permitted one to check whether shifting the test word during the saccade toward

DISTRIBUTION OF INITIAL LANDING POSITIONS
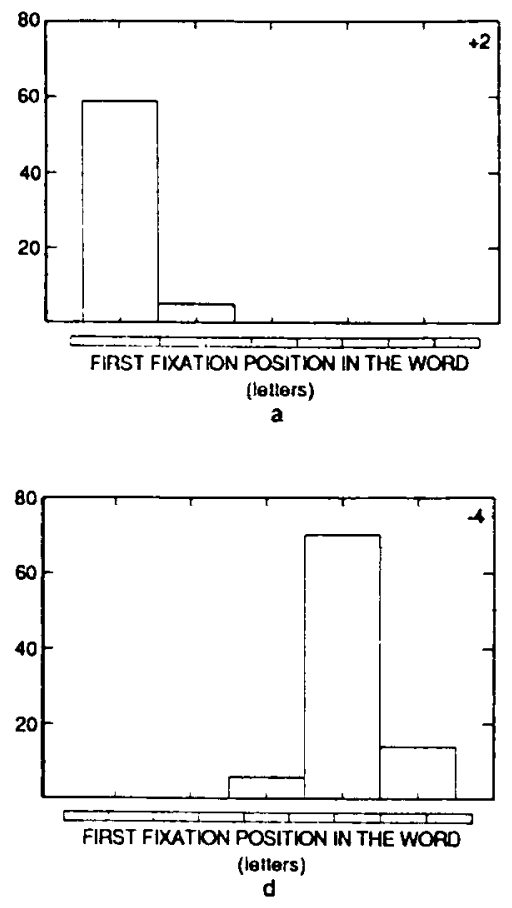
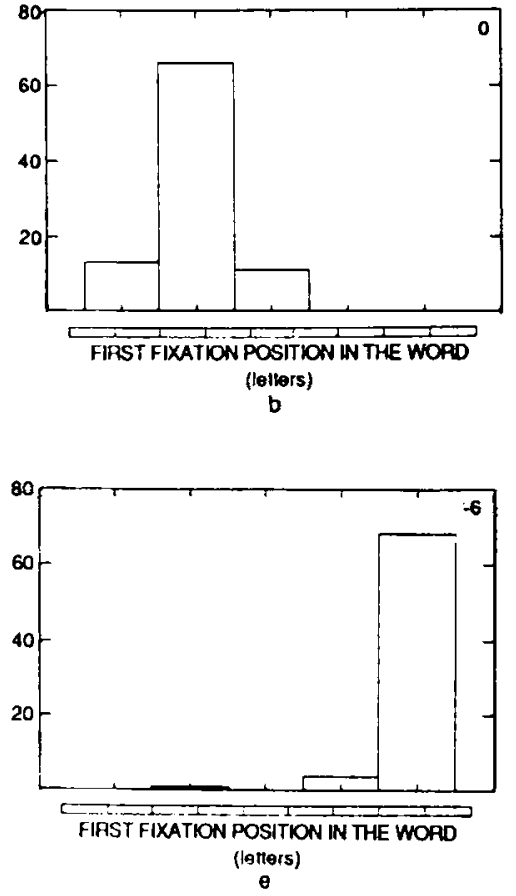

9- letter words of high frequency

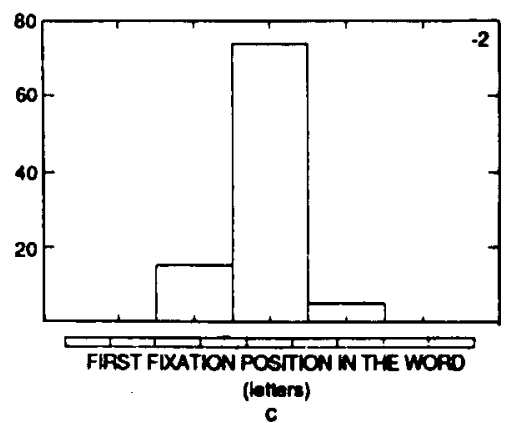

Figure 3. Example of the distribution of initial landing positions in the test word as a function of the amount of shifting $(+2,0,-2$, -4 , and -6 ), for 9-letter words of high frequency, in the condition with parafoveal preprocessing when the preceding context was predictable. 
this word had the expected effect and would permit an analysis of the pattern of fixations on the test word as a function of the eye's initial landing position. This analysis confirmed that when the test word was not shifted, the initial landing positions were located essentially near the middle for 5-letter words and left of the middle for 9-letter words (see, e.g., the distribution of initial landing positions for 9-letter words of high frequency, in Figure 3). Shifting the test word to the right shifted the distribution of initial landing positions leftward into the test word, and shifting it to the left shifted the distribution rightward. Thus, through division of the word into five equal zones, the proportion of data for each zone of the word appeared to be approximately equal.

Before the analysis of the fixation patterns on the test word as a function of the eye's initial fixation position in the word could be made, it was also necessary to check that shifting the test word did not in itself modify the within-word tactics or the individual durations of fixations on the test word. A first argument is that subjects were unaware of the extent of the display shift; because of the line of masking Xs that occurred during the saccade, a large perturbation was visible for all shifts (including no shift at all), rendering negligible any differences caused by shift extents. As a further control, the gaze durations (which correspond to the total time the eye spent on the word and which directly reflect the within-word tactics and individual fixation durations) obtained for each of the five possible initial landing zones with different shift amounts were compared. In Figures $4 \mathrm{a}$ and $4 \mathrm{~b}$ are plotted the mean gaze duration, for 5- and 9-letter words (all frequencies combined), for each landing zone, separately for each amount of shift, for all the conditions of parafoveal preprocessing and linguistic context combined. As is apparent from these figures, the segments of curves corresponding to different shift amounts are approximately superposed. Thus, it seems that shifting the test word did not affect the gaze duration. This conclusion is supported by further analyses.

For each possible initial landing zone, each word length and frequency, and each condition of linguistic context and parafoveal preprocessing, a Student's $t$ test was performed on mean gaze durations obtained with two different shift amounts (which were in most cases adjacent amounts, as, e.g., 0 and -1 , but which could also be more, as, e.g., 0 and -3 , when enough data were available for each shifting amount). In only $10 \%$ of the comparisons made was there an effect of the amount of shift, and in the remaining cases, there was no systematic pattern of dependency of the gaze duration differences on the amount of shift. Moreover, if we look again at the segments of the curves corresponding to each amount of shift in Figures $4 a$ and $4 b$, it appears that the gaze duration strongly depends on the eye's first fixation position, just as it does for the optimal landing position phenomenon, whatever the amount of shift. It thus is reasonable to assume that the optimal landing position effect and other
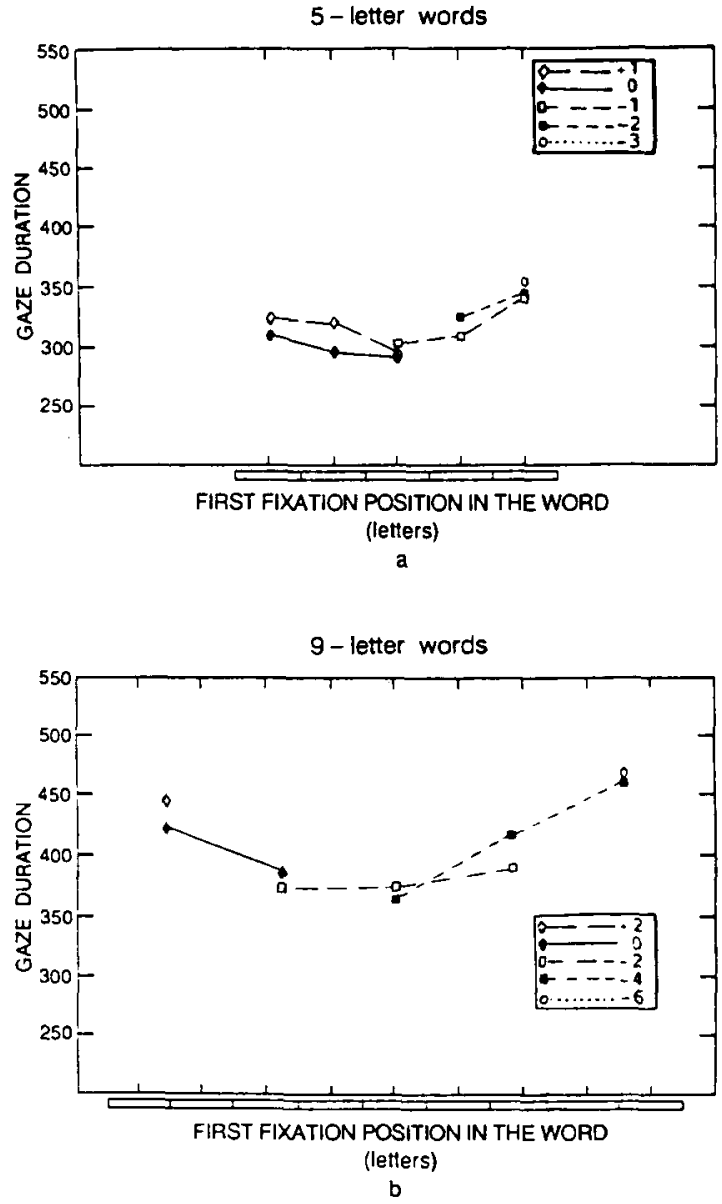

Figure 4. Gaze duration on the test word (the total time the eye spent on the word) as a function of the eye's initial landing position in the test word (five zones, corresponding to word length divided by five), for the different shifting amounts $(+1,0,-1,-2$, and -3 , for 5-letter words, and $+2,0,-2,-4$, and -6 , for 9-letter words) for 5- and 9-letter words (a and b, respectively); all the conditions of parafoveal preprocessing and linguistic context, as well as all word frequencies, are combined. Note that for each shifting amount, only the mean gaze duration calculated on more than $25 \%$ of the subjects is represented.

effects to be discussed were not caused by the sentence shifts themselves.

In order to estimate the influence of parafoveal preprocessing and linguistic context on the optimal landing position phenomenon, the strength of the effect of the eye's initial landing position on the refixation probability and gaze duration will be compared between the different conditions of parafoveal preprocessing and linguistic context. Statistically, these comparisons require examination of the interactions of parafoveal preprocessing and linguistic context with the initial fixation position, and so these will be presented first. The global effects of parafoveal preprocessing and linguistic context on refixation probability and gaze duration will be examined after that, in order 
to determine whether the results obtained in this experiment are comparable to those found in other studies in which initial fixation position was not manipulated.

\section{Did Parafoveal Preprocessing and}

Linguistic Context Affect the Strength of the Optimal Landing Position Effect?

If we consider first the probability of refixating the test word as a function of the eye's initial landing position in the word, we can observe that there is for all word lengths and frequencies an optimal landing position phenomenon, whatever the condition of parafoveal preprocessing and linguistic context; when the eye first fixated near the word's middle, the probability of refixating the word was lower than it was when the eye first fixated other positions (see Figures 5a-5d and 6a-d).

The analysis of variance confirmed this observation, since the effect of the eye's initial landing position in the test word on refixation probability was significant in all the conditions. When words were preceded by a neutral sentence, $F(4,76) \geq 6.7, p<.0005$, and $F(4,76) \geq 5.0$, $p<.0005$, for all word lengths and frequencies in the conditions without and with parafoveal preprocessing, respectively; and when words were predictable from preceding context, $F(4,76) \geq 7.1, p<.0005$, for all word lengths and frequencies in the condition without parafoveal preprocessing, and $F(4,76)=6.4, p<.0005, F(4,76)$ $=3.3, p<.025, F(4,76)=5.1, p<.005$, and $F(4,76)$ $=11.4, p<.0005$, for 5- and 9-letter words of low frequency and 5- and 9-letter words of high frequency, respectively, in the condition with parafoveal preprocessing.

The quadratic analysis of the curves showed the same pattern. When words were preceded by a neutral sentence, $F(1,19) \geq 17.1, p<.0005$, for all word lengths and frequencies in the condition without parafoveal preprocessing, and $F(1,19)=38.2, p<.0005, F(1,19)=$ $16.1, p<.001, F(1,19)=16.8, p<.001$, and $F(1,19)$ $=13.2, p<.005$, for 5 - and 9-letter words of low fre-
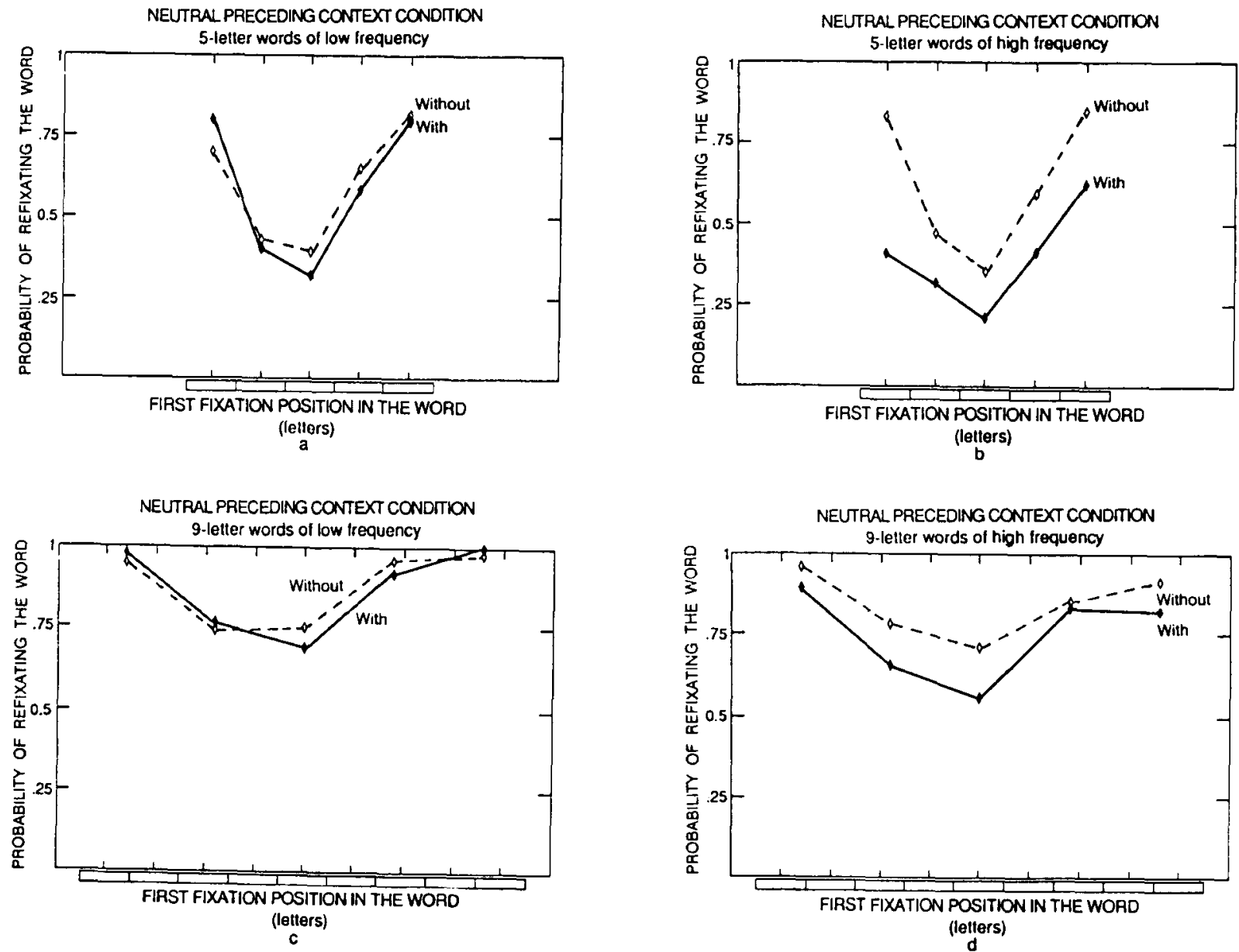

Figure 5. Probability of refixating the test word in the neutral preceding context condition, as a function of the eye's initial landing position in the test word in the condition with parafoveal preprocessing (solid lines) and in the condition without parafoveal preprocessing (dashed lines), for 5-letter words (of low [a] and high frequency [b]) and 9-letter words (of low [c] and high frequency [d]). For all word lengths, the middle of the abscissa corresponds to the middle of the word. 

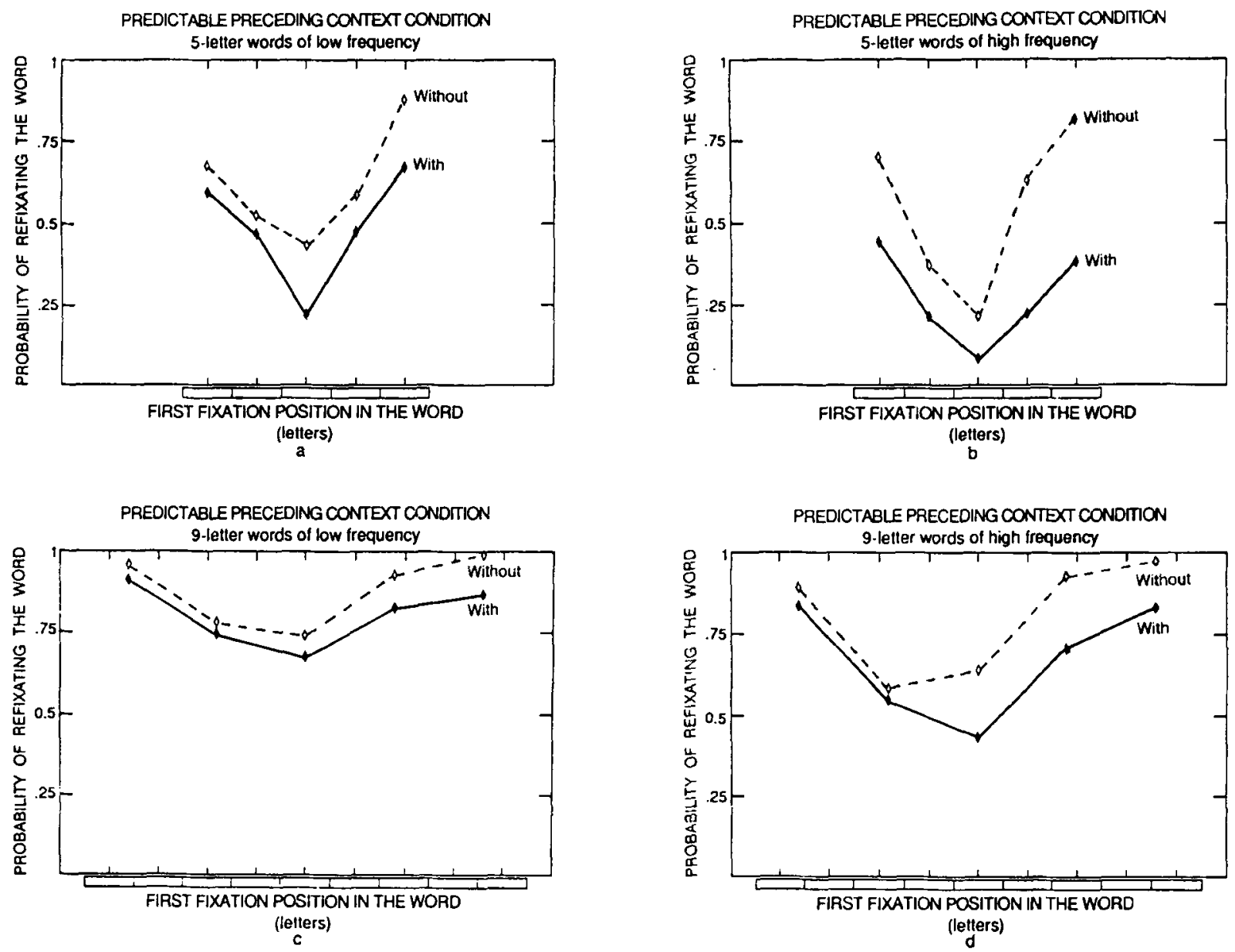

Figure 6. Probability of refixating the test word in the predictable preceding context condition, as a function of the eye's initial landing position in the test word, in the condition with parafoveal preprocessing (solid lines) and in the condition without parafoveal preprocessing (dashed lines), for 5-letter words (of low [a] and high frequency [b]) and 9-letter words (of low [c] and high frequency [d]).

quency and 5- and 9-letter words of high frequency, respectively, in the condition with parafoveal preprocessing; and when words were predictable from the preceding context, $F(1,19)=28.5, p<.0005, F(1,19)=16.0$, $p<.001, F(1,19)=17.2, p<.001$, and $F(1,19)=$ $45.4, p<.0005$, for 5- and 9-letter words of low frequency and 5- and 9-letter words of high frequency, respectively, in the condition without parafoveal preprocessing, and $F(1,19)=27.7, p<.0005, F(1,19)=7.2$, $p<.025, F(1,19)=28.5, p<.0005$, and $F(1,19)=$ $31.9, p<.0005$, for 5 - and 9-letter words of low frequency and 5- and 9-letter words of high frequency, respectively, in the condition with parafoveal preprocessing.

As can be seen from Figures 5a-5d and 6a-6d, parafoveal preprocessing did not change the strength of the optimal la:ding position effect whatever the preceding context condition; the slopes of the optimal landing position curves were approximately identical in both conditions of parafoveal preprocessing, except in the case of 5-letter words of high frequency, with which there was a slight weakening of the optimal landing position effect in the condition wiith parafoveal preprocessing. Although the three-way interaction between parafoveal preprocessing, linguistic context, and first fixation position was never significant $[F(4,76) \leq 2.513, p<.10$, for all word lengths and frequencies], the two-way interaction between parafoveal preprocessing and the first fixation position was significant in the case of 5-letter words of high frequency that were predictable from preceding context: In the neutral preceding context condition, $F(4,76)$ $=.729$, n.s., $F(4,76)=.748$, n.s., $F(4,76)=2.338$, $p<.10$, and $F(4,76)=.98$, n.s., for 5- and 9-letter words of low frequency and 5- and 9-letter words of high frequency, respectively, and in the predictable preceding context condition, $F(4,76)=.981$, n.s., $F(4,76)=.491$, n.s., $F(4,76)=3.154, p<.05$, and $F(4,76)=2.277$, $p<.10$, for 5- and 9-letter words of low frequency and 5- and 9-letter words of high frequency, respectively. The linguistic context alone did not affect the strength of the optimal landing position effect (see Figures 5a-5d and 
6a-6d). The interaction between preceding context and first fixation position in the condition without parafoveal preprocessing was never significant except in the case of 9-letter words of high frequency $[F(4,76)=.931$, n.s., $F(4,76)=.393$, n.s., $F(4,76)=1.333$, n.s., and $F(4,76)$ $=3.818, p<.01$, for 5- and 9-letter words of low frequency and 5- and 9-letter words of high frequency, respectively]. However, this significant interaction for 9letter words of high frequency probably resulted from the leftward shift of the optimal landing position in the case in which the preceding context was predictable relative to the case in which it was neutral.

As concerns the gaze duration on the test word as a function of the eye's initial landing position (see Figures $7 a-7 d)$, the same effect was found. When words were preceded by a neutral sentence, there was an optimal landing position (near the middle of words) in the conditions with and without parafoveal preprocessing, whatever the word length and the word frequency. In this case, the effect of the eye's initial landing position in the test word was always significant; for the global analysis, $F(4,76)$ $=5.0, p<.005, F(4,76)=3.0, p<.05, F(4,76)=$
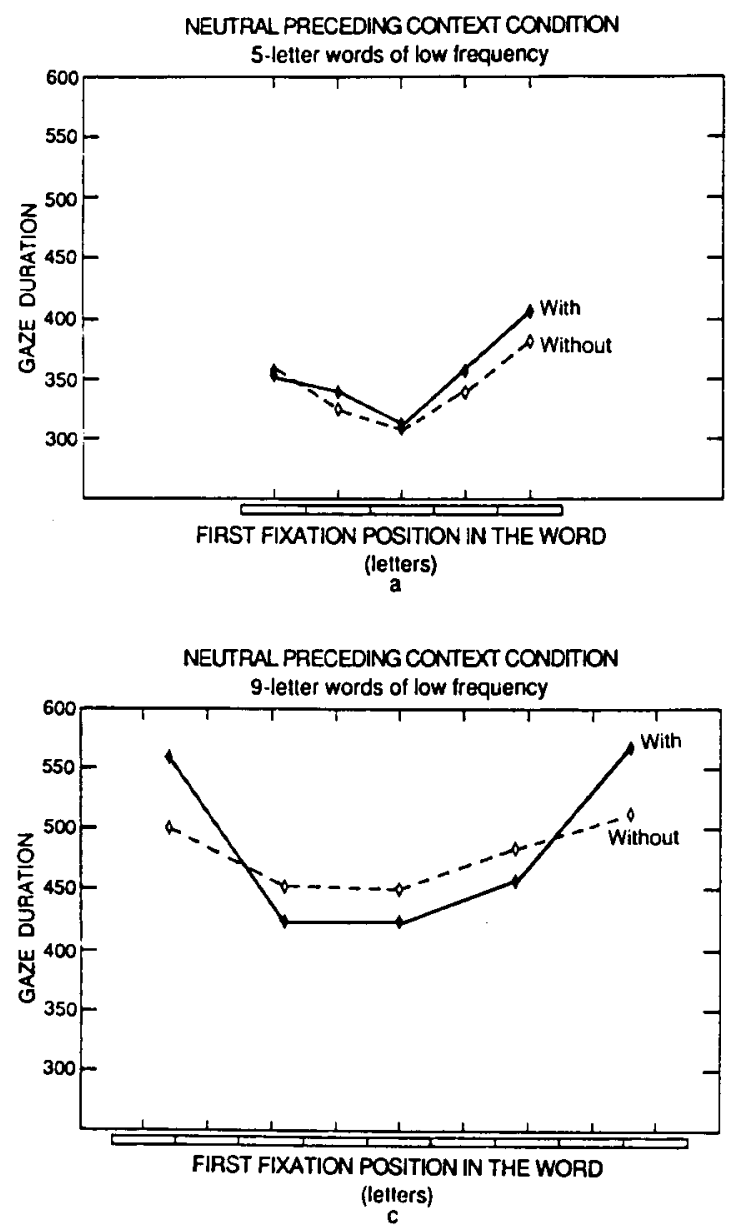

$4.5, p<.005$, and $F(4,76)=22.5, p<.0005$, for 5 and 9-letter words of low frequency, and 5- and 9-letter words of high frequency, respectively, in the condition without parafoveal preprocessing, and $F(4,76)=10.1$, $p<.0005, F(4,76)=11.4, p<.0005, F(4,76)=2.1$, $p<.10$, and $F(4,76)=5.9, p<.0005$, for 5- and 9letter words of low frequency and 5- and 9-letter words of high frequency, respectively, in the condition with parafoveal preprocessing; and for the quadratic analysis, $F(1,19)=20.9, p<.0005, F(1,19)=7.2, p<.025$, $F(1,19)=21.5, p<.0005$, and $F(1,19)=68.7, p<$ .0005 , for 5- and 9-letter words of low frequency and 5and 9-letter words of high frequency, respectively, in the condition without parafoveal preprocessing, and $F(1,19)$ $=32.7, p<.0005, F(1,19)=28.8, p<.0005, F(1,19)$ $=8.8, p<.01$, and $F(1,19)=16.5, p<.0005$, for 5- and 9-letter words of low frequency and 5- and 9-letter words of high frequency, respectively, in the condition with parafoveal preprocessing.

For words that were predictable from the preceding context, there was again an optimal landing position effect in both conditions of parafoveal preprocessing (see Fig-
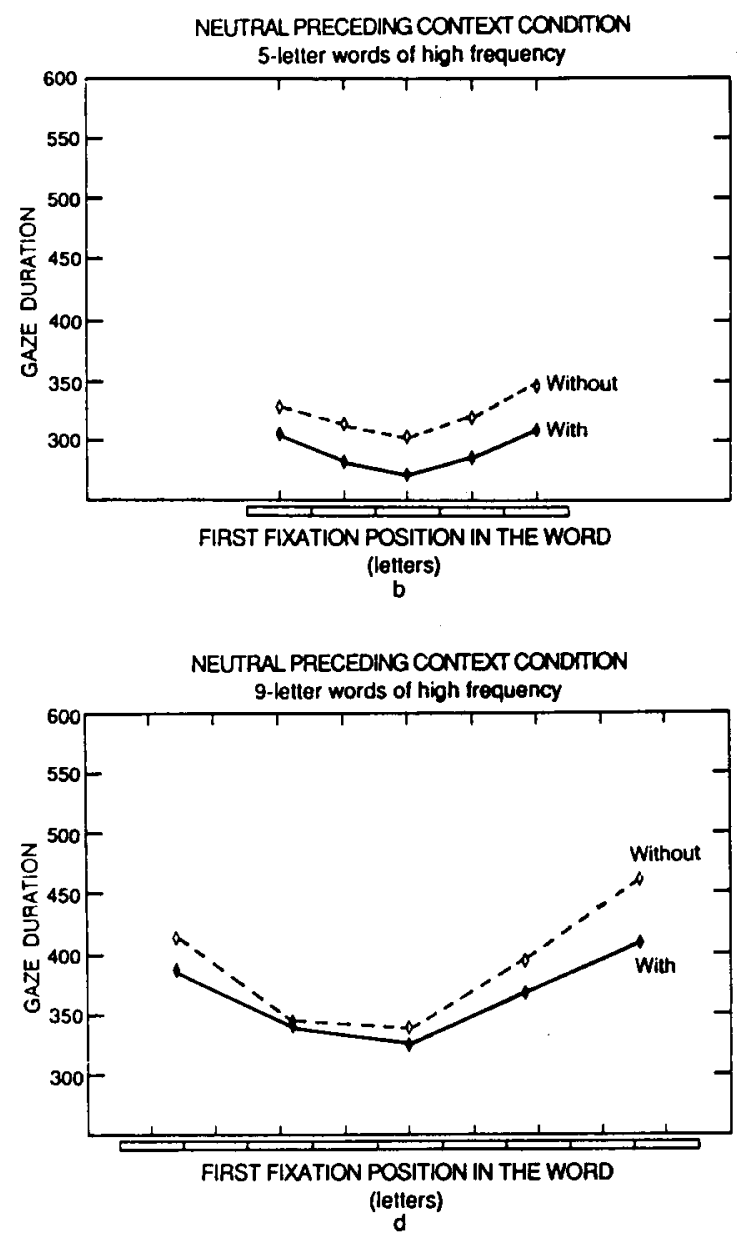

Figure 7. Gaze duration on the test word in the neutral preceding context condition, as a function of the eye's initial landing position in the test word, in the condition with parafoveal preprocessing (solid lines) and in the condition without parafoveal preprocessing (dashed lines), for 5-letter words (of low [a] and high frequency [b]) and 9-letter words (of low [c] and high frequency [d]). 
ures 8a-8d). However, although the effect of the eye's initial landing position in long words was always significant, this effect for short words was only significant in the condition without parafoveal preprocessing $[F(4,76)$ $=2.7, p<.05, F(4,76)=15.5, p<.0005, F(4,76)$ $=10.8, p<.0005$, and $F(4,76)=29.4, p<.0005$, for 5- and 9-letter words of low frequency and 5- and 9letter words of high frequency, respectively; $F(4,76)=$ 1.5 , n.s., $F(4,76)=5.3, p<.005, F(4,76)=.6$, n.s., and $F(4,76)=9.1, p<.0005$, for 5- and 9-letter words of low frequency and 5- and 9-letter words of high frequency, respectively, in the condition with parafoveal preprocessing]. The quadratic analysis of the curves revealed exactly the same pattern $[F(1,19)=5.7, p<.05$, $F(1,19)=66.3, p<.0005, F(1,19)=29.4, p<.0005$, and $F(1,19)=59.7, p<.0005$, for 5- and 9-letter words of low frequency and 5- and 9-letter words of high frequency, respectively, in the condition without parafoveal preprocessing, and $F(1,19)=1.4$, n.s., $F(1,19)=13.9$, $p<.005, F(1,19)=2.4$, n.s., and $F(1,19)=49.2$,
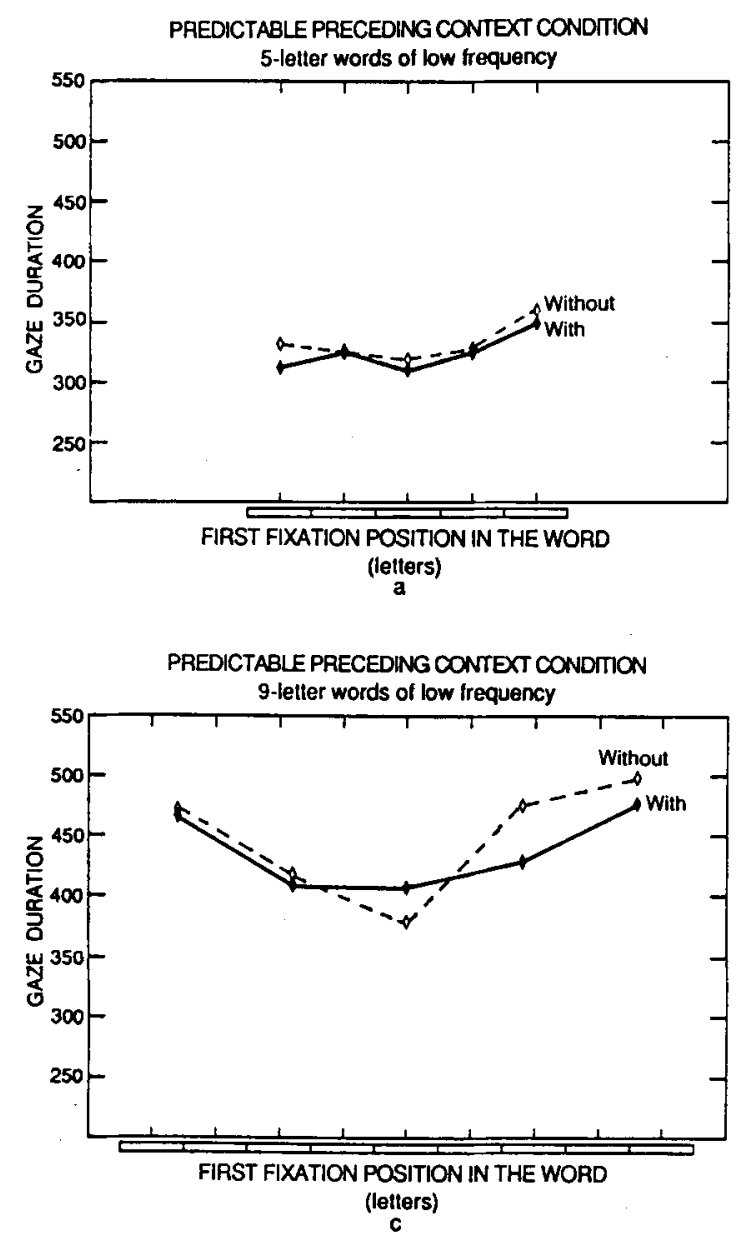

Figure 8. Gaze duration on the test word in the predictable preceding context condition, as a function of the eye's initial landing position in the test word, in the condition with parafoveal preprocessing (solid lines) and in the condition without parafoveal preprocessing (dashed lines), for 5-letter words (of low [a] and high frequency [b]) and 9-letter words (of low [c] and high frequency [d]). $p<.0005$, for 5- and 9-letter words of low frequency and 5- and 9-letter words of high frequency, respectively, in the condition with parafoveal preprocessing].

Parafoveal preprocessing seemed to decrease the strength of the optimal landing position effect only when words were predictable from preceding context. In the neutral preceding context condition, contrary to the predictions, this factor seemed to increase the optimal landing position effect in the case of low-frequency words. However, the three-way interaction between parafoveal preprocessing, linguistic context, and first fixation position was significant only in the case of long words of low frequency $[F(4,76)=.34$, n.s., $F(4,76)=3.16, p<$ $.05, F(4,76)=1.141$, n.s., and $F(4,76)=.15$, n.s., for 5- and 9-letter words of low frequency and 5- and 9-letter words of high frequency, respectively]. The two-way interaction between parafoveal preprocessing and the first fixation position was significant only in the case of 9-letter words of low frequency in the neutral preceding context condition and in the case of 9-letter words of high fre-
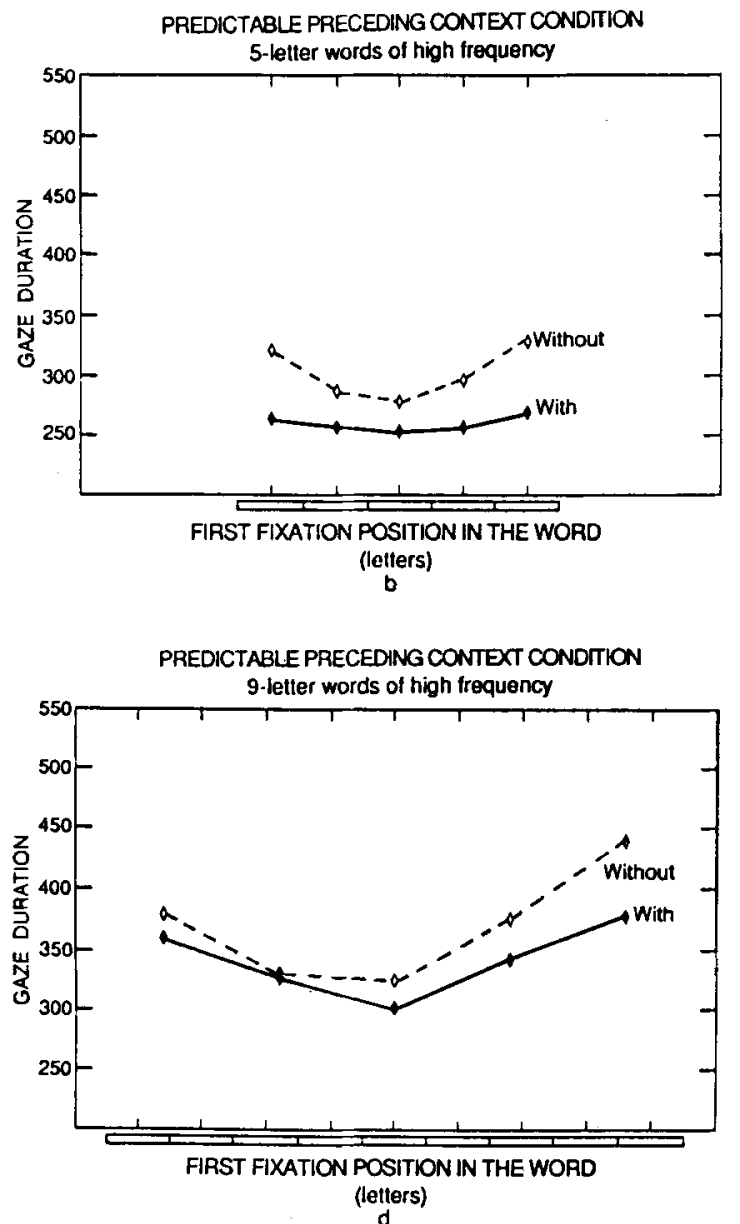

d 
quency in the predictable preceding context condition $[F(4,76)=.549$, n.s., $F(4,76)=3.108, p<.025$, $F(4,76)=.141$, n.s., and $F(4,76)=1.287$, n.s., for 5 and 9-letter words of low frequency and 5- and 9-letter words of high frequency, respectively, in the neutral preceding context condition, and $F(4,76)=.22$, n.s., $F(4,76)=2.247, p<.10, F(4,76)=2.35, p<.10$, and $F(4,76)=3.088, p<.025$, for 5- and 9-letter words of low frequency and 5- and 9-letter words of high frequency, respectively, in the predictable preceding context condition]. The interaction between preceding context and first fixation position in the condition without parafoveal preprocessing was never significant $[F(4,76)$ $=1.486$, n.s., $F(4,76)=1.273$, n.s., $F(4,76)=.771$, n.s., and $F(4,76)=.438$, n.s., for 5- and 9-letter words of low frequency and 5- and 9-letter words of high frequency, respectively].

Thus, from these results, it appeared that there was no systematic weakening of the optimal landing position effect in the presence of parafoveal preprocessing, linguistic context, or both factors combined, whether for refixation probability or for gaze duration.

\section{Did Peripheral Preprocessing and Linguistic Context Globally Influence Oculomotor Behavior?}

The absence of a systematic and significant effect of parafoveal preprocessing and linguistic context on the strength of the optimal landing position effect could result from the fact that these factors failed to globally influence refixation probability as well as gaze durations. In order to check for this possibility, the probability of refixating the test word and the gaze duration were examined, whatever the eye's initial landing position in the test word, as a function of the parafoveal preprocessing and preceding context conditions (these effects are illustrated in Figures 4a-4d and 5a-5d, for refixation probability, and in Figures $6 a-6 d$ and $7 a-7 d$, for gaze duration). The influence of parafoveal preprocessing and linguistic context on individual fixation durations was also studied, but this will not be developed in detail here. All of the analyses revealed differences between the different word frequencies.

For low frequency words, the probability of refixating the test word (see Figures 4 and 5) was smaller in the condition with parafoveal preprocessing than it was in the condition without parafoveal preprocessing, but only when the preceding context was predictable $[F(1,19)=8.73$, $p<.01$, and $F(1,19)=7.049, p<.025$, for 5 - and 9 letter words, respectively], the effect of parafoveal preprocessing being nonsignificant when the preceding context was neutral $[F(1,19)=.183$, n.s., $F(1,19)=.043$, n.s., for 5- and 9-letter words, respectively]. The interaction between parafoveal preprocessing and linguistic context was significant for 5-letter words $[F(1,19)=$ $5.584, p<.05]$ and only tended to be significant in the case of 9-letter words $[F(1,19)=3.566, p<.10]$.
Gaze duration was slightly smaller in the condition with parafoveal preprocessing than it was in the condition without parafoveal preprocessing, but only when words were predictable from the preceding context (see Figures 6 and 7). However, the effect of parafoveal preprocessing was nonsignificant in both conditions of preceding context. In the predictable preceding context condition, $F(1,19)=.427$, n.s., and $F(1,19)=.921$, n.s., for 5and 9-letter words, respectively, and in the neutral preceding context condition, $F(1,19)=.286$, n.s., and $F(1,19)=.1$, n.s., for 5- and 9-letter words, respectively. Moreover, the interaction between parafoveal preprocessing and linguistic context was nonsignificant $[F(1,19)=$ $3.045, p<.10, F(1,19)=1.818$, n.s., for 5- and 9-letter words, respectively]. This absence of an influence of parafoveal preprocessing probably resulted from the fact that parafoveal preprocessing did not affect individual fixation durations. The gaze duration when there was only one fixation on the test word was only slightly decreased with parafoveal preprocessing when words were predictable from preceding context. Moreover, the gaze duration when two fixations were made did not differ as a function of parafoveal preprocessing, whatever the condition of preceding context. Since the advantage in terms of refixation probability in the condition with parafoveal preprocessing relative to the condition without parafoveal preprocessing when words were predictable from preceding context was small (.14 for 5-letter words and .007 for 9 letter words), it was not sufficient to create a significant effect for gaze durations where variability resulting from variability of individual fixation durations is greater than the variability of the refixation probabilities.

For high-frequency words with preceding context, both the refixation probability and the gaze duration were smaller in the condition with parafoveal preprocessing than they were in the condition without parafoveal preprocessing. There was a significant effect of this factor on the refixation probability [in the predictable preceding context condition, $F(1,19)=54.141, p<.0005$, and $F(1,19)=15.659, p<.001$, for 5- and 9-letter words, respectively, and in the neutral preceding context condition, $F(1,19) \geq 17.689, p<.0005$, for all word lengths], and also on gaze duration [in the predictable preceding context condition, $F(1,19)=25.564, p<.0005$, and $F(1,19)=5.768, p<.05$, for 5- and 9-letter words, respectively, and in the "neutral preceding context condition, $F(1,19)=8.885, p<.01$, and $F(1,19)=7.88$, $p<.025$, for 5- and 9-letter words, respectively]. This influence of parafoveal preprocessing was approximately equal for both conditions of preceding context, since the interaction between parafoveal preprocessing and preceding context was nonsignificant [for refixation probability, $F(1,19) \leq 1.449$, n.s., for both word lengths, and for gaze duration, $F(1,19) \leq 1.885$, n.s., for both word lengths]. The effect of parafoveal preprocessing on gaze duration in both conditions of preceding context resulted from the effect of this factor not only on the probability 
of refixating the test word but also on the gaze duration when there was only one fixation on the test word. No effect of parafoveal preprocessing was found for gaze duration when there were two fixations on the test word.

The global effect of preceding context was also analyzed. For low-frequency words, preceding context decreased refixation probability only when parafoveal preprocessing was available. However, the effect of preceding context in this case was significant only for 5-letter words $[F(1,19)=6.403, p<.025$, and $F(1,19)=4.2$, $p<.10$, for 5- and 9-letter words, respectively]. When there was no parafoveal preprocessing, the effect of preceding context was never significant $[F(1,19) \leq .433$, n.s., for both word lengths]. Gaze durations were shorter in the predictable preceding context condition than in the neutral preceding context condition, for both conditions of parafoveal preprocessing. [In the condition with parafoveal preprocessing, $F(1,19)=8.526, p<.01$, and $F(1,19)=16.459, p<.001$, for 5 - and 9-letter words, respectively, and in the condition without parafoveal preprocessing, $F(1,19)=8.55, p<.01$, and $F(1,19)=9.9$, $p<.01$, for 5- and 9-letter words, respectively.] The fact that the preceding context influenced gaze durations in both conditions of parafoveal preprocessing, for all word lengths, whereas it affected refixation probability only in specific cases, shows that preceding context also affected individual fixation durations. There was a slight influence of preceding context on gaze durations when there were only one or two fixations on the test word.

For high-frequency words, the probability of refixating the test word was smaller in the predictable preceding context condition than it was in the neutral preceding context condition, for both conditions of parafoveal preprocessing. However, the analysis of variance revealed that when parafoveal preprocessing was available, the ef- fect of preceding context was significant for both 5- and 9-letter words $[F(1,19)=13.307, p<.0005$, and $F(1,19)=6.747, p<.025]$, whereas when there was no parafoveal preprocessing, the effect of preceding context was significant only for 5-letter words $[F(1,19)=$ 8.676, $p<.01$, and $F(1,19)=4.302, p<.10$ ]. Gaze duration was systematically shorter in the predictable preceding context condition than it was in the neutral preceding context condition. In the condition with parafoveal preprocessing, $F(1,19)=19.566, p<.0005$, and $F(1,19)=12.725, p<.005$, for 5- and 9-letter words, respectively, and in the condition without parafoveal preprocessing, $F(1,19)=21.141, p<.0005$, and $F(1,19)$ $=13.825, p<.005$, for 5- and 9-letter words, respectively.] Preceding context also affected individual fixation durations; the gaze duration when there were only one or two fixations on the test word was shorter in the predictable preceding context condition than it was in the neutral preceding context condition.

\section{DISCUSSION}

\section{On the Weakening of the Optimal Landing Position Effect during Reading}

Many experiments have revealed the existence of an optimal landing position in words when they are presented either in isolation or within texts: When the eye first fixates near the middle of words, the probability of refixating these words, as well as the gaze duration, is shorter than it is when the eye begins to fixate other positions in words (McConkie et al., 1989; O'Regan \& LévySchoen, 1987; O'Regan et al., 1984; Vitu et al., 1990). A recent experiment in which the strength of the optimal landing position phenomenon was compared in both situations has shown that this effect is strongly weakened
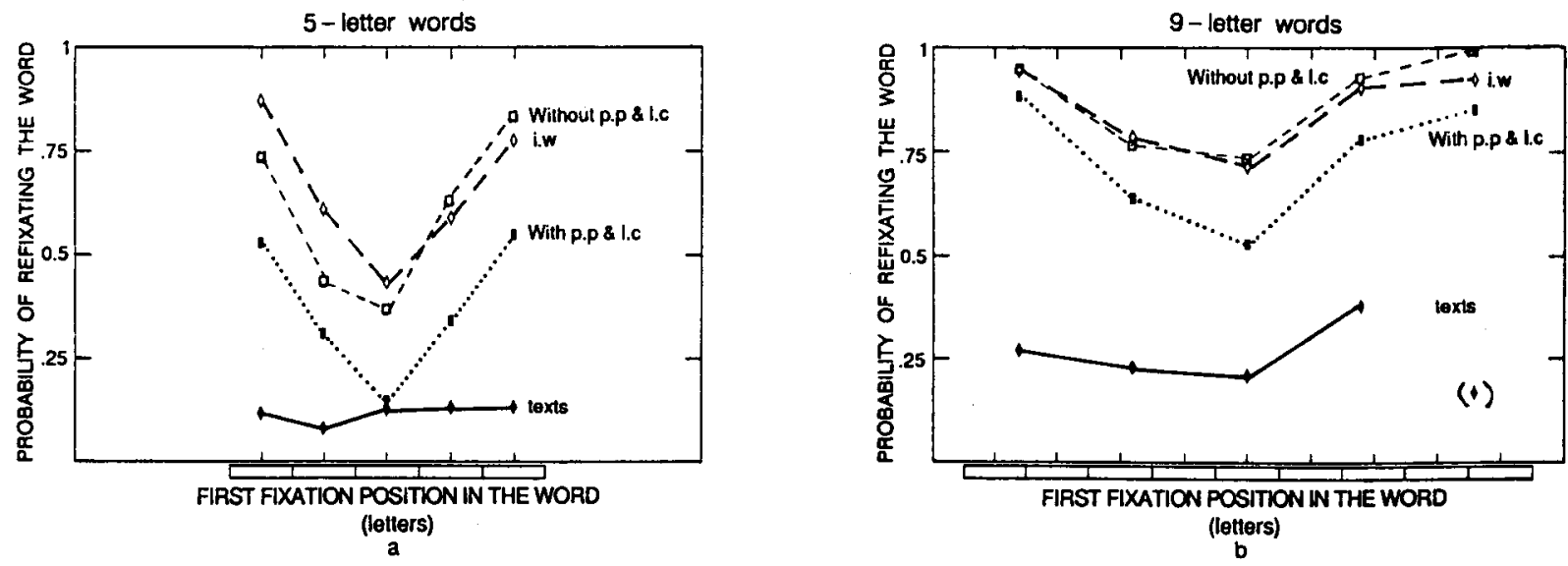

Figure 9. Comparison of the optimal landing position effect obtained for 5- and 9-letter words in two different conditions in the present experiment (one in which words could not be predicted from preceding context or preprocessed in parafoveal vision, and one in which linguistic context and parafoveal preprocessing were available) with the optimal landing position effect obtained by Vitu, ORegan, and Mittau (1990), for 5- and 9-letter words, during isolated word recognition and text reading. The graph represents the probability of refixating the test word as a function of the eye's initial landing position in the test word in the four situations that are named: without parafoveal preprocessing and linguistic context, with parafoveal preprocessing and linguistic context, isolated word, and text. 
for refixation probabilities and almost canceled for gaze durations in the text reading situation relative to the isolated word reading situation (Vitu et al., 1990).

In order to understand the reason for this weakening, the present experiment tested the influence on the optimal landing position phenomenon of two factors specific to the text reading situation that are known to facilitate word recognition and to affect oculomotor behavior: parafoveal preprocessing and linguistic context. The results showed that the effect of the eye's initial landing position in words on the refixation probability was equally strong with or without parafoveal preprocessing, as well as with predictable or with neutral preceding context-except in the case of 5-letter words of high frequency, for which a slight weakening was observed when both factors were available. These results are summarized in Figure 9, which presents the data corresponding to both extreme conditions of reading words: one in which they cannot be predicted or preprocessed in parafoveal vision, and one in which both linguistic context and parafoveal preprocessing are available. In Figure 9, it can be seen that these additional factors do not change the slopes of the optimal landing position curves. Since text reading differs from isolated word recognition at least in terms of the fact that words are predictable from preceding context and can be preprocessed in parafoveal vision, it seems that the weakening of the optimal landing position in text reading relative to isolated word reading cannot result from the influence of these factors. This conclusion will also be reached if we compare the curves with those obtained by Vitu et al. (1990), since the slopes of the curve obtained in the presence of both factors seem to fit better to that obtained in isolated word recognition than to that obtained with text reading by Vitu et al. (1990).

A possible explanation for why neither linguistic context nor parafoveal preprocessing was found to weaken the optimal landing position effect should be considered. This is the slowness of the reading created by the recording constraints in the present experiment. Although one would expect a priori that a longer-than-usual delay between context and test word should if anything have increased the chances that context and parafoveal preprocessing would be able to act, it is conceivable that for some reason the slight unnaturalness of the task prevented normal reading procedures from operating. If this were true, however, context and parafoveal preprocessing should not have had a global effect on gaze duration and probability of refixation on the test words; yet, as will be seen below, they did have such an effect, and this effect was comparable to that found by other authors in situations more like normal reading (e.g., Balota et al., 1985). Moreover, the fact that the analysis done a posteriori on the text reading data obtained by Vitu et al. (1990) showed no effect of parafoveal preprocessing on the optimal landing position phenomenon seems to add evidence that the weakening of this phenomenon during text reading does not result from the influence of such a factor, even when it is reinforced by linguistic context.

Thus the weakening of the optimal landing position during text reading must be due to the influence of other factors specific to text reading. One possibility might be the fact that during reading the eye is engaged in a sequence of forward saccades that pull the eye always farther into the text. Such a reading rhythm could decrease the probability of refixating the word even when the eye was not at the best place in the word to process it. This reading rhythm would not have been active in the present experiment because isolated sentences rather than continuous texts were used, and because of the particular presentation conditions that were involved, with delays longer than are usual in reading. Another related possibility is that during text reading, it might not be so important as it is
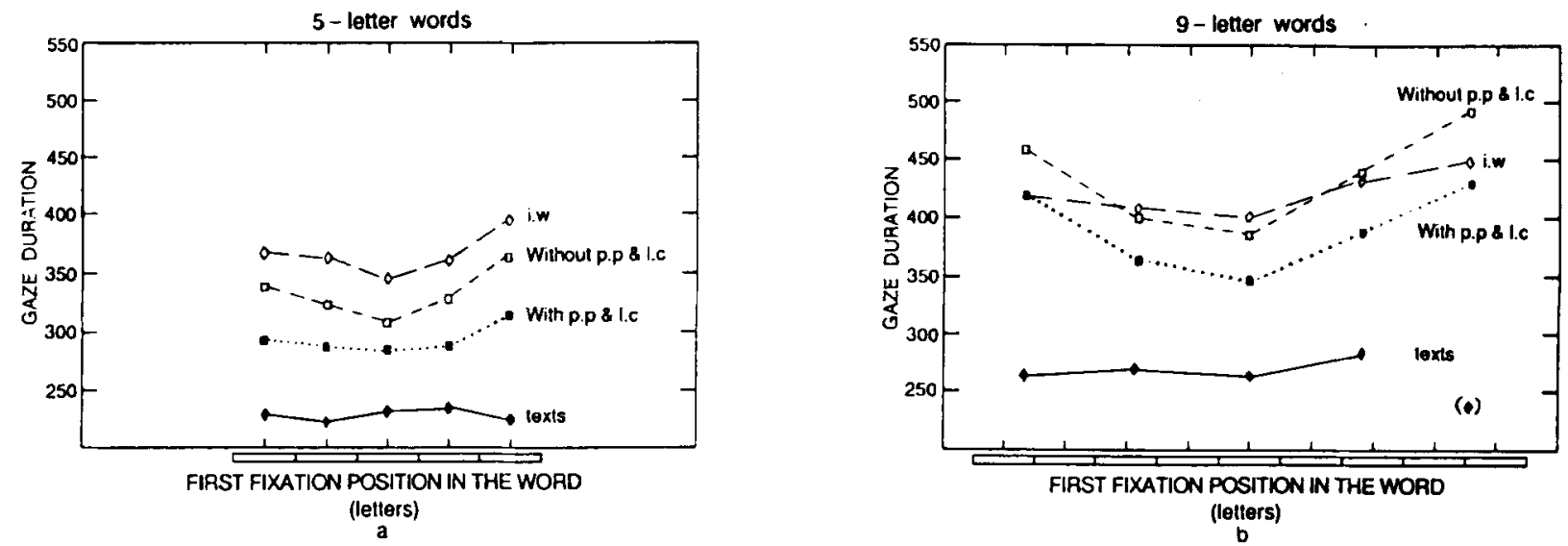

Figure 10. The optimal landing position effect for 5- and 9-letter words, obtained, on the one hand, in the present experiment in two different conditions (one in which words could not be predicted from preceding context or preprocessed in parafoveal vision, and one in which linguistic context and parafoveal preprocessing were available), and, on the other hand, during isolated word recognition and text reading in Vitu, O'Regan, and Mittau (1990). The graph represents the gaze duration on the test word, as a function of the eye's initial landing position in the test word, in the four situations that are named: without parafoveal preprocessing and linguistic context, with parafoveal preprocessing and linguistic context, isolated word, and text. 
during isolated word recognition to extract and process all the visual information before leaving the test word. These possibilities must be tested in future experiments.

Whereas parafoveal preprocessing and linguistic context did not affect the optimal landing position effect for refixation probability (except in the case of 5-letter words of high frequency, for which a slight weakening could be observed), these factors when they were combined seemed to weaken this phenomenon for gaze duration, at least in the case of short words (see Figure 10). Even though the analysis of variance showed no interaction between parafoveal preprocessing, linguistic context, and the first fixation position for this class of words for gaze duration, the effect of the eye's initial landing position in the word was nonsignificant when these words could be preprocessed in parafoveal vision and predicted by the preceding context, whereas it was significant in all the other conditions (see results section). This observation indicates that individual fixation durations are more variable in the presence of parafoveal preprocessing and linguistic context, rendering the effect of the eye's initial fixation position in the word on gaze duration less significant. This conclusion has also been reached by Vitu et al. (1990), who found a greater weakening of the optimal landing position phenomenon for gaze duration than that which could be predicted from the results concerning refixation probability. However, since in the present experiment no weakening was observed for gaze duration in the case of 9-letter words in the presence of parafoveal preprocessing and linguistic context, it seems that the weakening (for gaze duration) of the optimal landing position effect during text reading does not result only from these factors. Such a conclusion can be reached again by comparison of the results obtained in this experiment and the results in Vitu et al. (1990) for text reading (see Figure 10). One of the other factors specific to the text reading situation that could be responsible for the weakening of the optimal landing position phenomenon is the reading rhythm, which could affect both refixation probabilities and individual fixation durations.

\section{What Determines Within-Word Tactics and Gaze Duration on a Word?}

The absence of an influence of parafoveal preprocessing and linguistic context on the strength of the optimal landing position effect does not mean that these factors can be neglected in a model of oculomotor control during reading. The global effects of these factors, which were observed in this experiment independently of the eye's initial landing position in the test word, constitute evidence that they are as essential as the eye's initial landing position. This is why it is important to discuss the main and interactive effects of parafoveal preprocessing and linguistic context, which appeared to differ as a function of word frequency and as a function of the measure of the oculomotor behavior that was considered.

To summarize, as concerns refixation probabilities, on the one hand, when words were of low frequency, para- foveal preprocessing and linguistic context interacted in such a way that each factor alone could not decrease the probability of refixating the test word, but when they were combined, an effect was present. On the other hand, when words were of high frequency, parafoveal preprocessing and linguistic context had additive effects. As concerns gaze durations, when words were of low frequency, no interaction between parafoveal preprocessing and linguistic context was found; parafoveal preprocessing did not affect the gaze duration, whatever the condition of preceding context, and preceding context systematically decreased the gaze duration (this was true in all conditions of parafoveal preprocessing). When words were of high frequency, parafoveal preprocessing and linguistic context again had additive effects.

In the same way, if we look at the effects of word frequency, we can see that the refixation probability is significantly smaller for high-frequency words than for lowfrequency words, for all word lengths and in all conditions, except in the case in which there was no parafoveal preprocessing or linguistic context (see Figures 11a-11b).

\section{Word frequency effect 5-letter words}

PROBABILITY OF REFIXATING THE TEST WORD

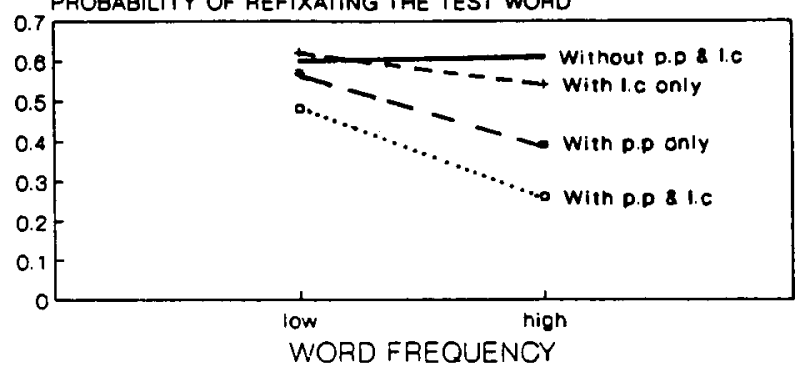

a

\section{9-letter words}

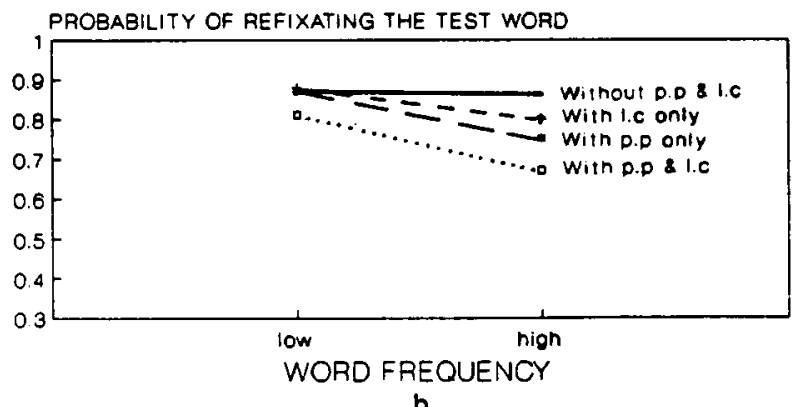

b

Figure 11. Probability of refixating the test word as a function of word frequency, in the four different situations used in the present experiment (without parafoveal preprocessing and linguistic context, with parafoveal preprocessing only, with linguistic context only, with parafoveal preprocessing and linguistic context), for 5-letter words (a) and 9-letter words (b). The data obtained for the different initial landing zones were meaned. 
Furthermore, gaze duration is significantly shorter for high-frequency words than it is for low-frequency words, whatever the word length and the conditions of parafoveal preprocessing and linguistic context, with word frequency and context having additive effects (see Figures 12a-12b).

At a first glance, some of the results of the present experiment could seem to contradict some of the data presented in the literature. First, Balota et al. (1985) found an effect of parafoveal preprocessing alone on refixation probability and gaze duration, whereas such an effect was observed here only in the case of high-frequency words. However, their data concerned words that could be classified relative to our materials as words of medium frequency. Second, Inhoff and Rayner (1986) showed that parafoveal preprocessing decreases gaze duration for both high- and low-frequency words, the size of the effect being identical for the two classes of words. However, again the word frequencies used by the authors were globally higher than they were in the present experiment.

As concerns the word-frequency effect, the results obtained here are compatible with Rayner and Duffy's

\section{Word frequency effect 5-letter words}

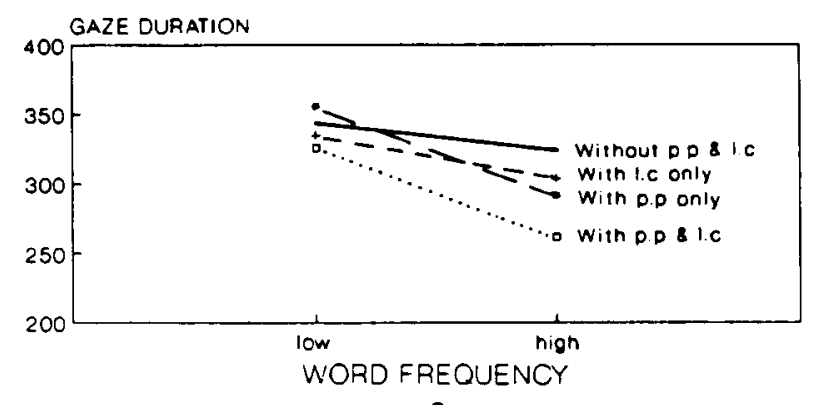

a

\section{9-letter words}

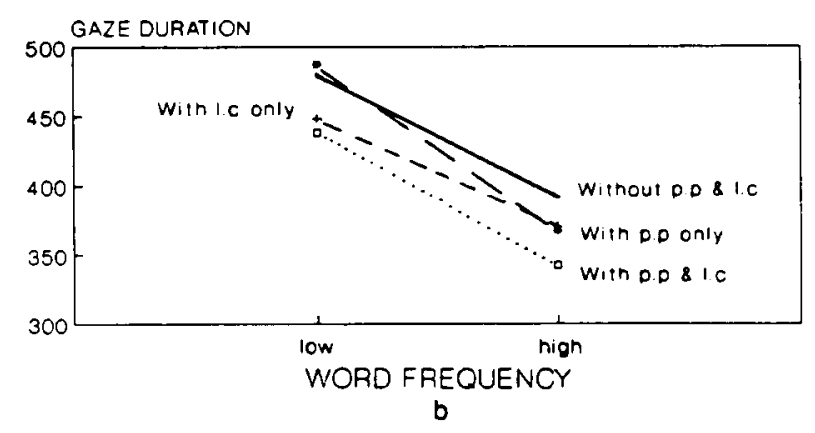

Figure 12. Gaze duration on the test word as a function of word frequency, in the four different situations used in the present experiment (without parafoveal preprocessing and linguistic context, with parafoveal preprocessing only, with linguistic context only, with parafoveal preprocessing and linguistic context), for 5-letter words (a) and 9-letter words (b). The data obtained for the different initial landing zones were meaned.
(1986) and Inhoff and Rayner's (1986) findings that word frequency affects gaze duration. They are also compatible with the data from Vitu et al. (1990) that showed an effect of word frequency on refixation probability only in the case of text reading, and an effect of word frequency on gaze duration in both text reading and isolated word recognition. Moreover, the effects of word frequency on gaze duration reproduce findings from Schuberth and Eimas (1977) that linguistic context and word frequency have additive effects during a lexical decision task.

The results of the present experiment are interesting for several reasons. First, the interactive effects of parafoveal preprocessing, linguistic context, and word frequency on the refixation probability show that the decision to refixate a word is dependent on the processing done on the word and that it cannot be determined only, as O'Regan and Lévy-Schoen (1987) and O'Regan (1990) supposed, by a preestablished program based on the eye's initial fixation position in the word. Second, since context and word frequency can separately affect gaze duration, whereas they had to be combined together or with parafoveal preprocessing to influence refixation probability, it seems that the word processing continues after the decision to refixate the word is made. On the basis of these elements, we could suppose that the following sequence of events takes place during the word scanning. When the eye lands on the word, visual processing begins and progresses with time. When a given delay is passed, the system examines the level of the processing that has been done on the word. Two cases could then be observed. If the level of completed processing is superior to a given amount that has been determined previously and that is probably less than the recognition threshold, the eye would directly leave the word without refixating it. In the other case, if the processing done on the word is not sufficiently advanced at the required delay, the word would be examined in two fixations. In both cases, the eye would leave the test word when the processing of the word would end or at least when a final processing threshold previously determined would be attained.

The fact that parafoveal preprocessing, linguistic context, and word frequency have to be combined to reduce the probability of refixating the word could indicate that the decision concerning the destination of the next saccade is made early during the fixation, probably at a moment when the visual information extracted is partial. Thus, in order that the word not be refixated, much information supplementary to the visual information would be necessary for one to attain the required preliminary threshold in the required delay. In other words, the advantage caused by parafoveal preprocessing, linguistic context, or word frequency would be insufficient to complete efficiently the visual information, and two or more of these factors would have to act together to prevent a refixation. On the contrary, since the visual processing of the fixated stimulus improves with time, after the decision to refixate the word is made, only one factor would be sufficient to facilitate the word processing over the fi- 
nal threshold. This could explain why linguistic context and word frequency can separately affect gaze duration. The absence of an influence of parafoveal preprocessing alone on gaze duration could result from the fact that the effect of this factor is transient; after a certain time, the prime created by the presentation of the word in parafoveal vision would no longer be available.

Further analyses (not presented here), which unfortunately were done on few observations, are compatible with these hypotheses concerning the time course of the influence of parafoveal preprocessing, linguistic context, and word frequency. Parafoveal preprocessing decreased gaze duration when there was only one fixation on the word, but only when it was reinforced by other factors (linguistic context and word frequency). This factor never affected gaze duration when exactly two fixations occurred on the word. In the same way, linguistic context and word frequency decreased gaze duration in the case of one fixation, but only when they were combined together or with another advantage. However, linguistic context and word frequency systematically affected the gaze duration in the case of two fixations.

All these results seem to indicate that the advantages caused by the possibility of preprocessing a word in parafoveal vision or of predicting it from the preceding context and the advantage caused by the presentation of a high-frequency word are available early during word processing. Thus, following McClelland and O'Regan (1981) on the one hand, and McClelland and Rumelhart's (1981) model of word recognition on the other, we could suppose that each of these factors affects the resting level of activation of the unit in the lexicon that is associated with the presented word. It is true that the precise locus of the effect of these factors cannot be determined on the basis of the results obtained in the present experiment. However, the fact that they all act at the same time cannot be explained by models of word recognition like that proposed by Paap, Newsome, McDonald, and Schvaneveldt (1982), in which the word frequency is supposed to play a role during word processing only if the prediction made on the basis of the contextual information is not good enough for one to recognize the word.

Since the effect of the eye's initial fixation position on refixation probability is the same whatever the conditions of parafoveal preprocessing and linguistic context and whatever the word frequency, it probably affects different stages of word processing than these factors. The hypothesis proposed here is that the first fixation position in the word determines the efficiency of the visual processing done on the word. Nazir, O'Regan, and Jacobs (1991) have shown that the amount of visual information extracted from the letters in the word is greater when the eye fixates near the middle of the word than when it fixates in the extreme parts of the word. Thus, when the eye begins to fixate near the optimal landing position of a word, the visual processing of the word would be faster than when it initially fixates other parts in the word, and the probability that the processing will attain the prelimi- nary recognition threshold in the required delay would be larger, thereby preventing a refixation of the word.

\section{CONCLUSION}

In order to understand the reason for the weakening of the optimal landing position effect during text reading relative to isolated word recognition, the influence of parafoveal preprocessing and linguistic context on this phenomenon were tested. Although the results show no effect of these factors on the strength of the optimal landing position effect, they do show that these factors strongly affect the refixation probability when they are combined together or when the presented word is of high frequency. These results seem to indicate that the decision to refixate a word is not determined by a preestablished program based only on the eye's initial landing position in the word, but that it directly depends on the amount of word processing done at a given moment.

\section{REFERENCES}

Balota, D. A., \& RAYNer, K. (1983). Parafoveal visual information and semantic contextual constraints. Journal of Experimental Psychology: Human Perception \& Performance, 9, 726-738.

Balota, D. A., Pollatsek, A., \&ayner, K. (1985). The interaction of contextual constraints and parafoveal visual information in reading. Cognitive Psychology, 17, 364-390.

Carroll, P., \&lowiczek, M. L. (1986). Constraints on semantic priming in reading: A fixation time analysis. Memory \& Cognition, $14,509-522$.

Duffy, S. A., Henderson, J. M., Morris, R. K. (1989). Semantic facilitation of lexical access during sentence processing. Joumal of Experimental Psychology: Leaming, Memory, \& Cognition, 15, 791-801.

EHrLICH, S. F., RAYNER, K. (1981). Contextual effects on word perception and eye movements during reading. Joumal of Verbal Leaming \& Verbal Behavior, 20, 641-655.

Fischler, I. S., BLOOM, P. A. (1979). Automatic and attentional processes in the effect of sentence context on word recognition. Journal of Verbal Learning \& Verbal Behavior, 18, 1-20.

Fischler, I. S., \& BLOOM, P. A. (1985). Effects of constraint and validity of sentence contexts on lexical decisions. Memory \& Cognition, 13, 128-139.

Holmes, V. M., \& O'Regan, J. K. (1987). Decomposing french words. In J. K. O'Regan \& A. Lévy-Schoen (Eds.), Eye movements: From physiology to cognition (pp.459-466). Amsterdam: Elsevier North-Holland.

INHOFF, A. W. (1989). Parafoveal processing of words and saccade computation during eye fixations in reading. Journal of Experimental Psychology: Human Perception \& Performance, 15, 544-555.

INHOFF, A. W., RAYNER, K. (1986). Parafoveal word processing during eye fixations in reading: Effects of word frequency. Perception \& Psychophysics, 40, 431-439.

LimA, S. D., \& INHOFF, A. W. (1985). Lexical access during eye fixations in reading: Effects of word-initial letter sequence. Journal of Experimental Psychology: Human Perception \& Performance, 11, 272-285.

McClelland, J. L., \& O'Regan, J. K. (1981). Expectations increase the benefit derived from parafoveal visual information in reading words aloud. Joumal of Experimental Psychology: Human Perception \& Performance, 7, 634-644.

MCClelland, J. L., \& Rumelhart, D. E. (1981). An interactive activation model of context effects in letter perception: Part 1 . An account of basic findings. Psychological Review, 88, 375-407.

McConkie, G. W., Underwood, N. R., Zola, D., \& Wolverton, G. S. (1985). Some temporal characteristics of processing during read- 
ing. Joumal of Experimental Psychology: Human Perception \& Performance, 11, 168-186.

McConkie, G. W., KerR, P. W., Reddix, M. D., Zola, D., \& JACOBs, A. M. (1989). Eye movement control during reading: ПI. Frequency of refixating a word. Perception \& Psychophysics, 46, 245-253.

MEYer, D. E., \& SChVANEVELDT, R. W. (1971). Facilitation in recognizing pairs of words: Evidence of a dependence between retrieval operations. Joumal of Experimental Psychology, 90, 227-234.

Nazir, T. A., O'Regan, J. K., \& Jacobs, A. M. (1991). On words and their letters. Bulletin of the Psychonomic Society, 29, 171-174.

O'ReGAN, J. K. (1984). How the eye scans isolated words. In A. G. Gale \& E. Johnson (Eds.), Theoretical and applied aspects of eye movement research: Proceedings of the European Conference on Eye Movements (pp. 159-168). Amsterdam: Elsevier North-Holland.

O'ReGAN, J. K. (1989). Visual acuity, lexical structure, and eye movements in word recognition. In B. Elsendoorn \& H. Bouma (Eds.), Working models of human perception (pp. 261-292). London: Academic Press.

O'RegaN, J. K. (1990). Eye movements and reading. In E. Kowler (Ed.), Reviews of oculomotor research: Vol. 4. Eye movements and their role in visual and cognitive processes (pp. 395-453). Amsterdam: Elsevier.

O'Regan, J. K., \& LÉvy-Schoen, A. (1987). Eye movement strategy and tactics in word recognition and reading. In M. Coltheart (Ed.), Attention and performance XII: The psychology of reading (pp. 363383). Hillsdale, NJ: Erlbaum.

O'Regan, J. K., Lévy-Schoen, A., Pynte, J., \& Brugaillère, B. (1984). Convenient fixation location within isolated words of different length and structure. Journal of Experimental Psychology: Human Perception \& Performance, 10, 250-257.

PaAP, K. R., Newsome, S. L. (1981). Parafoveal information is not sufficient to produce semantic or visual priming. Perception \& Psychophysics, 29, 457-466.

PaAp, K. R., Newsome, S. L., McDonald, J. E., Schvaneveldt, R. W. (1982). An activation-verification model for letter and word recognition: The word-superiority effect. Psychological Review, 89, 573-594.

RAYNER, K. (1978). Foveal and parafoveal cues in reading. In J. Requin (Ed.), Attention and performance VII (pp. 149-161). Hillsdale, NJ: Erlbaum.

RAYNER, K., \& DUFFY, S. A. (1986). Lexical complexity and fixation times in reading: Effects of word frequency, verb complexity, and lexical ambiguity. Memory \& Cognition, 14, 191-201.

Rayner, K., McConkie, G. W., Ehrlich, S. (1978). Eye movements and integrating information across fixations. Journal of $E x$ - perimental Psychology: Human Perception \& Performance, 4, 529-544.

RAYNeR, K., MCCONKIE, G. W., \& Zola, D. (1980). Integrating information across eye movements. Cognitive Psychology, 12, 206-226.

Ross, L. E., \& Ross, S. M. (1980). Saccade latency and warning signals: Stimulus onset, offset, and change as warning events. Perception \& Psychophysics, 27, 251-257.

SCHUBERTH, R. E., Eimas, P. D. (1977). Effects of context on the classification of words and non-words. Joumal of Experimental Psychology: Human Perception \& Performance, 3, 27-36.

Schwanenflugel, P. J., Shoben, E. J. (1985). The influence of sentence constraint on the scope of facilitation for upcoming words. Joumal of Memory \& Language, 24, 232-252.

Simpson, G. B., Peterson, R. R., Casteel, M. A., Burgess, C. (1989). Lexical and sentence context effects in word recognition. Journal of Experimental Psychology: Leaming. Memory, \& Cognition, 15, 88-97.

Stanovich, K. E., \& West, R. F. (1979). Mechanisms of sentence context effects in reading: Automatic activation and conscious attention. Memory \& Cognition, 7, 77-85.

STANOVICH, K. E., \& WEST, R. F. (1981). The effect of sentence context on ongoing word recognition: Tests of a two-process theory. Journal of Experimental Psychology: Human Perception \& Performance, 7, 658-672.

Stanovich, K. E., \& West, R. F. (1983). On priming by a sentence context. Journal of Experimental Psychology: General, 112, 1-36.

VITU, F. (in press). The existence of a global effect during reading. Vision Research.

VITU, F., \& O'RegAN, J. K. (1988). Effect of parafoveal preprocessing and reading thythm on optimal landing position in words of different length and frequency. In G. Luer, U. Lass, \& J. Schallo-Hoffmann (Eds.), Eye movement research: Physiological and psychological aspects (pp. 286-292). Toronto, NY: Hogrefe.

VITU, F., \& O'ReGAN, J. K. (1990). Eye movement in reading: Optimal landing position and usefulness of peripheral preview. In D. Brogan (Ed.), The First International Conference on Visual Search Proceedings. London, NY: Taylor \& Francis.

Vitu, F., O'Regan, J. K., Mittau, M. (1990). Optimal landing position in reading isolated words and continuous texts. Perception \& Psychophysics, 47, 583-600.

ZoLA, D. (1984). Redundancy and word perception during reading. Perception \& Psychophysics, 36, 277-284.

(Manuscript received July 3, 1990; revision accepted for publication February 5, 1991.) 